\title{
Los trastornos de la personalidad en drogodependientes desde la perspectiva de los cinco grandes factores
}

\author{
Eduardo J. Pedrero Pérez \\ Psicólogo. Máster en Drogodependencias. CAD Sector IV. Plan Municipal contra las Drogas. Ayuntamiento de Madrid. \\ Enviar correspondencia a: \\ Eduardo J. Pedrero Pérez. C/ Bergantín, 11 5 B. 28042 - MADRID. Tfno: 609587233. ejpedrero@mi.madritel.es / ejpedrero@yahoo.es
}

\section{RESUMEN}

Objetivo: Explorar las relaciones entre las dimensiones básicas de personalidad, propuestas por el modelo de cinco factores y medidas a través del Big Five Questionnaire (BFQ), y los trastornos de personalidad, estimados dimensional y categorialmente mediante el Millon Clinical Multiaxial Inventory II (MCMIII) en sujetos que se encuentran en tratamiento por abuso o dependencia de sustancias psicoactivas.

Material y método: La muestra está compuesta por 187 sujetos que se encuentran realizando tratamiento ambulatorio por abuso o dependencia de heroína, cocaína, alcohol, cannabis o benzodiazepinas, y que cumplimentan ambos cuestionarios simultáneamente. Se efectúan pruebas de correlación bivariada entre las dimensiones y subdimensiones del BFO y las escalas de trastornos de personalidad del MCMI-II. Se efectúa posteriormente un análisis de regresión de las dimensiones y subdimensiones del BFO sobre cada una de las escalas del MCMI-II.

Resultados: Se constata la especial implicación de las dimensiones de Estabilidad Emocional (Neuroticismo) y Energía (Eje Intraversión/Extraversión) en la mayor parte de los desórdenes, si bien cada uno de ellos presenta un patrón peculiar de organización del que participan los cinco rasgos, coherente con lo descrito en otras investigaciones similares y con la formulación teórica de cada trastorno. Finalmente se presentan gráficamente aquéllas relaciones que, según un criterio restrictivo, son confirmadas por ambos métodos, comparándose los resultados con los obtenidos en otros estudios similares.

Discusión: Se argumenta la implicación de estos resultados en la práctica clínica, así como en una posible clasificación dimensional, en el futuro, de los trastornos del Eje II. Se discuten, también, las limitaciones de las clasificaciones y los instrumentos utilizados para la evaluación de estos trastornos.

Conclusiones: Los trastornos de personalidad pueden ser caracterizados a partir de peculiares configuraciones de rasgos, lo que además de tener repercusiones para los sistemas clasificatorios, es de especial relevancia para el diseño de intervenciones psicoterapéuticas y farmacológicas.

Palabras clave: Personalidad, Trastornos de Personalidad, EvaIuación Clínica, Adicción, Modelo de Cinco Factores, BFQ, MCMI-II.

\section{SUMMARY}

Objective: To explore the relations between the basic dimensions of personality proposed by the five factors model, and measured with the Big Five Questionnaire (BFQ), and personality disorders, considered dimensionally and categorically with the Millon Clinical Multiaxial Inventory II (MCMI-II) in subjects that are in treatment for psychoactive substance abuse or dependency.

Material and method: The sample comprises 187 individuals undergoing outpatient treatment for heroin, cocaine, alcohol, cannabis or benzodiazepine abuse or dependency, and who complete both questionnaires simultaneously. Bivariate correlation was assessed between the BFQ dimensions and subdimensions and the MCMI-II personality disorder scales. Subsequently, we made a regression analysis of the BFO dimensions and subdimensions on each one of the MCMI-II scales.

Results: The particular implication of the dimensions of Emotional Stability (Neuroticism) and Energy (Introversion/Extraversion Axis) is seen in most of the disorders, although each one of them displays a peculiar pattern of organization in which all of the five characteristics participate, in line with what has been described in other similar research work, and with the theoretical formulation of each disorder. Finally, there is a diagram of those relationships that, according to a restrictive criterion, are confirmed by both methods, the results being compared with those from similar studies.

Discussion: The implication of these results is argued in relation to clinical practice, in addition to a possible dimensional classification, in the future, of the Axis II disorders. We also discuss the limitations of the classifications and the instruments used in the evaluation of these disorders.

Conclusions: Personality disorders can be characterized on the basis of peculiar configurations of traits which, as well as having repercussions on the classificatory systems, has particular relevance for the design of psychotherapeutic and pharmacological interventions.

Key words: Personality, Personality Disorders, Clinical Assessment, Addiction, Five Factor Model, BFQ, MCMI-II. 


\section{INTRODUCCIÓN}

E estudio de los trastornos de personalidad (en adelante, TTPP) ha adquirido un gran auge en las dos últimas décadas, sin que los resultados de la investigación hayan conseguido despejar las brumas en torno a un concepto tan controvertido. Queda fuera de toda duda que algunas personas presentan patrones estables de comportamiento que generan malestar al propio sujeto o a quienes le rodean, pero no está clara la verdadera naturaleza de tales patrones de conducta. La responsabilidad de tal indefinición hay que buscarla, como señalan Pelechano y cols. (1995) en el hecho de que la formulación de los trastornos de la personalidad en psicopatología se ha hecho al margen del desarrollo de la psicología de la personalidad, optándose por acentuar el consenso entre científicos en busca de un eclecticismo compartido, a riesgo de confundir validez de consenso social con el resto de valideces (de constructo, predictiva, concurrente, de contenido, diferencial, etc.). Consecuencia de ello es que la etiqueta "trastorno de personalidad" ha venido siendo aplicada, sin apellidos, a procesos difíciles de entender y de manejar, no encajables en las categorías diagnósticas disponibles o que carecen de un tratamiento conocido, lo que ha dotado al concepto de una innegable connotación peyorativa (Tyrer et al., 1993). No es extraño que algunos autores consideren a los TTPP como "los hijos bastardos de la psiquiatría" (Hirschfeld, 1993).

Todo lo dicho anteriormente supone, en primer lugar, la crisis de los modelos categoriales, incapaces, en el estado de conocimiento actual, de dar cuenta de manera adecuada de los problemas objeto de estudio: las categorías del Eje II no concuerdan con la evidencia empírica, no son entidades discretas sino solapadas entre sí, presentan criterios diagnósticos redundantes, etc. (Livesley, 2000; Westen y Shedler, 2000). El modelo biológico de enfermedad mental no ha sido capaz de dar cuenta de los denominados TTPP y en el intento de clasificarlos se han vulnerado, más que nunca, los principios básicos en que se apoyan las clasificaciones biológicas: que las categorías sean exhaustivas y mutuamente excluyentes (Blashfield, 1986). Y los sucesivos intentos por incrementar la fiabilidad de las categorías diagnósticas han tenido un relativo éxito pero a costa de un menosprecio hacia la validez (Meehl, 1989; Nelson-Gray, 1991), hasta el punto de que el constructo de trastorno de personalidad llega a ser, en sí mismo, una creencia ligada a la cultura que refleja los sesgos locales de la sociedad occidental (Lewis-Fernández y Kleinman, 1995). O como señalaba Millon (1991), tales sistemas de clasificación llevan aparejados otros problemas: 1) contribuyen a la falaz creencia de que los procesos psicopatológicos incluyen entidades discretas cuando de hecho éstas sólo son meros conceptos útiles para coordinar nuestras observaciones; 2) plantean la duda de si las categorías son artificiales o derivadas empíricamente, existiendo siempre el riesgo de imponer a unos datos una estructura que tal vez no exista; 3 ) no aciertan a identificar o incluir aspectos de la conducta, ante la necesidad de restringir la lista de atributos a un conjunto de características predeterminadas, con la consiguiente pérdida de información; 4) obligan a enfrentarse con el dilema de desarrollar criterios diagnósticos restrictivos para incrementar la homogeneidad en los miembros correspondientes a cada clase, a costa de tener que añadir un determinado número de categorías mixtas o "cajones de sastre" para incluir a un elevado número de sujetos que no logran cumplir dichos criterios; y 5) ponen de relieve que el número y diversidad de categorías suele ser muy inferior al de las diferencias individuales apreciadas en la práctica diaria (citado en Lemos, 1995).

Otra perspectiva más fructífera comienza a imponerse en los últimos años: a medida que los TTPP pasan a ser observados desde los hallazgos de la psicología de la personalidad, empieza a atisbarse una más adecuada conceptuación. De especial interés son los acercamientos que se han producido desde el modelo de los Cinco Grandes Factores. Este enfoque se caracteriza por una particular concepción de la ciencia (empirismo), una particular metodología (análisis factorial), un particular modelo estructural (rasgos dimensionales) y una particular tradición investigadora (hipótesis léxica), y propugna que cualquier tipo de personalidad puede ser conceptuada a partir de cinco amplias dimensiones ortogonales (Millon y Davies, 1998). En términos generales este modelo evidencia, mediante el análisis factorial, una solución de cinco factores que tiende a repetirse, aunque no está claro aún si con ese número se agota el número total de dimensiones de la personalidad a considerar o si todas las dimensiones poseen el mismo poder explicativo (Pelechano et al., 1995). Diversos estudios han puesto de manifiesto la gran estabilidad temporal de las medidas obtenidas mediante este modelo (Costa y Mc Crae, 1988), su consistencia y replicabilidad y su aplicabilidad (De Raad, 1994; Hogan, 1987; Wiggins y Pincus, 1992) y la posibilidad de generalizarlo a diversos entornos culturales (Avia et al., 1995; Borkenau y Ostendorf, 1993; Caprara y Perugini, 1994; Triandis y Eunkook, 2002).

No está clara aún la verdadera entidad de los cinco factores, pero empieza a acumularse evidencia empírica de que su existencia está ligada tanto a factores genéticos como ambientales. Los datos más recientes apuntan a que el $40 \%$ de la varianza de estos rasgos de personalidad son aportados por factores genéticos y que es el ambiente el que proporciona el $60 \%$ restante (Borkenau et al., 2001; Plomin et al., 1997). La estructura de personalidad basada en los cinco factores parece claramente fundamentada en 
ambos aspectos, siendo superior a modelos de más o de menos factores, como los de Cloninger y Eysenck respectivamente, incluso cuando se estudian muestras de diversas culturas (McCrae et al., 2001). La investigación se centra en la actualidad en la identificación de la parte atribuible a factores genéticos y la que únicamente se fundamenta en elementos ambientales, de cada uno de los rasgos, así como en la expresión fenotípica de los efectos combinados de ambos (Jang et al., 2001)

En los últimos años se ha prestado especial interés a la relación entre estas cinco grandes dimensiones y los trastornos de la personalidad (Sánchez Bernardos, 1995; Widiger y Frances, 2002b; Widiger y Sanderson, 1995; Widiger et al., 1994), en la hipótesis de que éstos puedan ser explicados en gran medida a partir de aquéllos, encontrándose que tal modelo predice mejor que otros la severidad de los trastornos (Ball, 2002). De hecho, se trabaja en la actualidad sobre la hipótesis de un futuro DSM-V en el que el Eje Il sería dimensional, de modo que se diagnosticarían uno o varios TTPP indicando en cada uno de ellos el grado de severidad observado (Oldham y Skodol, 2000; Westen y Shedler, 2000; Widiger et al., 2002a), siendo los rasgos identificados en el modelo de cinco factores las unidades estructurales cuya combinación en presencia y magnitud darían cuenta de los patrones desadaptativos que se categorizan como trastornos (Trull y McCrae, 2002).

El modelo de cinco factores no es el único que apoya el enfoque dimensional de los TTPP. Millon y su teoría bio-social (Millon y Davies, 1998) o la teoría de siete factores de Cloninger (Cloninger et al., 1993) investigan la personalidad y sus desordenes desde la metodología dimensional, aunque desde perspectivas sensiblemente diferentes. En concreto Millon, desde un eclecticismo psicológico y una visión biologicista de la génesis de los patrones desadaptativos de la personalidad, procura un acercamiento entre los enfoques dimensionales y los categoriales a partir de una metodología a medio camino entre lo racional y lo empírico. Cloninger, más próximo al modelo de cinco factores, propone una más amplia taxonomía incluyendo los componentes biológicos implicados en la etiología de los trastornos. En todo caso, parece incuestionable en el momento actual la superioridad de las aproximaciones dimensionales aunque es necesaria una mayor acumulación de investigaciones que demuestren empíricamente las ventajas de tal perspectiva (Nathan y Langenbucher, 1999; Widiger, 2000).

Especial interés reviste el tema con relación a determinadas poblaciones específicas, como la de los adictos a sustancias. Parece incuestionable, a tenor de la investigación disponible, que los sujetos que abusan o dependen de drogas presentan una prevalencia extremadamente alta de trastornos de la personalidad (Blume, 1989; Brooner, et al., 1997; Calsyn et al., 1996; Flynn et al., 1996; Halikas et al., 1994; Kosten et al., 1989) que alcanzan en algunos casos el $61 \%$ (DeJong et al., 1993) e incluso más (Kokkevi y Stefanis, 1995; Kosten y Rounsaville, 1986; Kranzler et al., 1994; Marlow et al., 1995). En nuestro país las cifras son algo menores, oscilando entre el 36\% (García y Ezquiaga, 1992) y el 53\% (Cervera et al., 1997), aunque algunos estudios encuentran muestras con prevalencias sensiblemente más bajas (Santos et al., 2001) y otros más elevadas (San Narciso et al., 2000; Sánchez et al., 1999) al evaluar subpoblaciones específicas. Cuando la evaluación se ha llevado a cabo utilizando instrumentos concebidos desde una perspectiva dimensional la prevalencia ha resultado aún mayor (Craig y Weiberg, 1992; Flynn et al., 1995; McMahon y Richards, 1996; Mestre et al., 2001; Nadeau et al., 1999).

De entre todos los patrones de personalidad disfuncional, los más relacionados con el consumo de sustancias son aquéllos que se basan en dimensiones de impulsividad, lo que puede ser entendido a partir de la vulnerabilidad para consumir sustancias, sin medir el riesgo que significa su uso, y caer en la adicción tras el consumo (Bolinches et al., 2002). Se ha estudiado la relación entre los trastornos de la personalidad, los trastornos del control de los impulsos y la adicción a drogas, llegándose a estimar que los tres conceptos comparten un sustrato conceptual común necesitado de una más amplia teoría que los relacione (Cervera et al., 2001). No obstante, la impulsividad no agota las relaciones entre las diversas personalidades y la autoadministración de sustancias, de modo que puede estimarse la funcionalidad de todas ellas frente a rasgos generales o particulares de cualquier patrón de comportamiento.

Algunos trabajos han abordado las relaciones entre los instrumentos de medida de los TTPP y los cuestionarios de rasgos elaborados desde el modelo de los Cinco Grandes Factores en población adicta. Brooner y cols. (1993) utilizaron el NEO-PI sobre una muestra de 203 adictos a opiáceos dividiéndolos en grupos según hubieran recibido un diagnóstico simple de trastorno antisocial, un diagnóstico mixto de éste y algún otro trastorno de personalidad y un grupo sin diagnóstico en el Eje II; destacaba la significación de la dimensión de Neuroticismo, que era mayor cuantos más diagnósticos se presentaban, sucediendo lo mismo aunque en menor magnitud con la dimensión de Afabilidad y no encontrándose diferencias significativas en el resto de factores. El estudio, de carácter longitudinal, es revisado con posterioridad (Brooner et al., 2002) encontrándose relaciones entre Neuroticismo, Afabilidad y Tesón, así como la subescala de Búsqueda de Sensaciones del factor Extraversión, con los diagnósticos del Eje II, resultados que confirman las relaciones obtenidas en otros estudios (Widiger et al., 2002c) y encontrando el alto potencial predictivo de 
respuesta al tratamiento a partir del modelo de cinco factores.

EI NEO-PI (Costa y McCrae, 1985) y su versión revisada el NEO-PI-R (Costa y McCrae, 1992) no son los únicos instrumentos utilizados desde el modelo de cinco factores. También se ha utilizado el Big Five Questionnaire (BFQ; Caprara et al., 1995) para estudiar la estructura de la personalidad de los drogodependientes (Berrocal et al., 2001; Pedrero, 2002) y su relación con los trastornos del Eje II en pacientes de Salud Mental (Iglesias et al., 2000).

El Inventario Clínico Muitiaxial de Millon II (Millon Clínical Muitiaxial Inventory-II, MCM-II, adaptación española de Ávila-Espada, Jiménez y colaboradores, 1999) es un instrumento concebido para explorar los trastornos de la personalidad desde una perspectiva dimensional que, además, ha sido adaptado para proporcionar una adecuación a los modelos categoriales. Su sistema clasificatorio de referencia es el DSM-III-R, aunque ya está disponible la versión MCMI-III que lo hace a partir del DSM-IV, si bien no disponemos aún de estudios de validez en población española, trabajo que es realizado en la actualidad por un equipo de la Universidad Complutense de Madrid.

\section{OBJETIVO}

Explorar la relación entre las cinco dimensiones básicas de la personalidad propugnadas por el modelo de los cinco grandes factores y los patrones de personalidad desadaptativa estimados a partir del modelo dimensional de Millon, en sujetos que se encuentran en tratamiento por abuso o dependencia de sustancias psicoactivas.

\section{MÉTODO}

\section{Participantes}

La muestra está compuesta por 187 sujetos que se encuentran en tratamiento por sus problemas con las drogas en un Centro de Atención a Drogodependencias del Ayuntamiento de Madrid (CAD-4) adscrito al Plan Municipal contra las Drogas de Madrid.

\section{Instrumentos}

Entrevista de Valoración Inicial, estructurada y estandarizada para todos los usuarios del servicio, que recoge datos sociodemográficos, legales, médicos, historia de consumo, historia de tratamiento por drogas o por problemas de salud mental y motivación para la solicitud de tratamiento. De ella se extraen, a los efectos de este estudio, las variables sexo, edad, droga que motiva la demanda, edad de inicio de consumo y tiempo de consumo de la sustancia principal.

Big Five Questionnaire (BFQ; Caprara et al., 1993; versión española de Bermúdez, 1995), cuestionario de autoinforme basado en el modelo de cinco factores, validado para población española y ofrece la posibilidad de presentar los resultados en puntuaciones T, según una distribución normal con media 50 y desviación típica 10, considerándose muy bajos los valores entre 25 y 34, bajos los comprendidos entre 35 y 44, promedio entre 45 y 54 , altos entre 55 y 64 y muy altos entre 65 y 75. Se compone de 132 items que se responden en una escala de Likert de 5 opciones, e informa de cinco dimensiones, cada una de ellas con dos subdimensiones: Energía (Dinamismo y Dominancia), Afabilidad (Cooperatividad y Cordialidad), Tesón (Escrupulosidad y Perseverancia), Estabilidad Emocional (Control de las Emociones y de los Impulsos) y Apertura Mental (Apertura a la Cultura y a la Experiencia); además de una escala de Distorsión (Ver Gráfico 1).

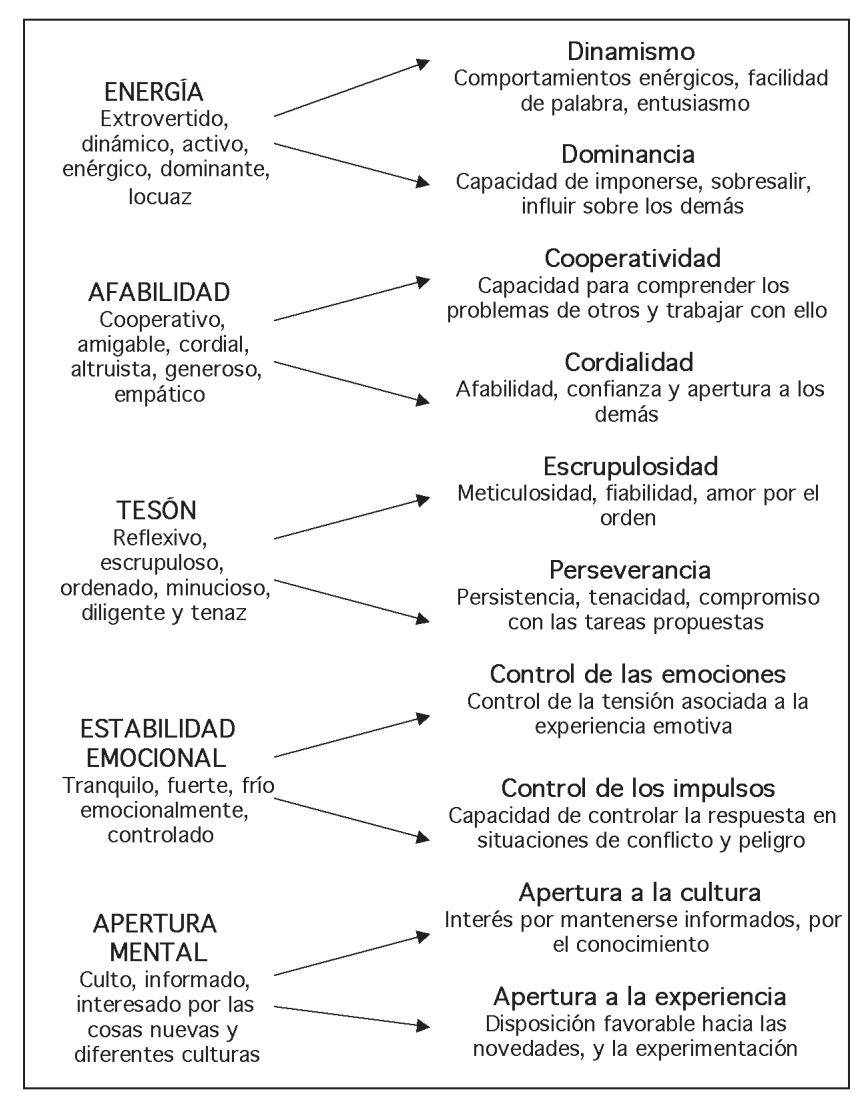

Gráfico 1. Dimensiones y subdimensiones del BFQ y rasgos fundamentales que describen.

El MCMI-II (Millon, 1999) es un cuestionario que consta de 175 items de respuesta verdadero-falso que informan sobre 8 patrones clínicos de personalidad, 3 formas graves de patología de personalidad, 6 síndromes clínicos de intensidad moderada y 3 síndromes clínicos graves. Las puntuaciones directas, por una 
parte, permiten la descripción dimensional de los diversos patrones desadaptativos y, por otra, se convierten en puntuaciones de tasa base atendiendo a la distribución de la prevalencia de cada trastorno en los subgrupos de población; la lógica de esta transformación se sitúa en el hecho de que, a diferencia de los rasgos, que se distribuyen normalmente y con frecuencias comparables, los trastornos de personalidad no presentan una distribución semejante ni su prevalencia es igual entre las poblaciones de pacientes, lo que obliga a transformar las puntuaciones atendendiendo al modelo de prevalencia subyacente a cada trastorno. Una vez considerado éste, mediante estudios poblacionales preliminares, el autor elabora unas tablas para cada subgrupo de población, estimando un punto de corte en el valor 75, siendo los valores iguales o superiores sugestivos de algún problema clínico, y otro en el valor 85 que indicaría una severidad importante del trastorno. El cuestionario cuenta con diversas medidas de validez, deseabilidad y sinceridad.

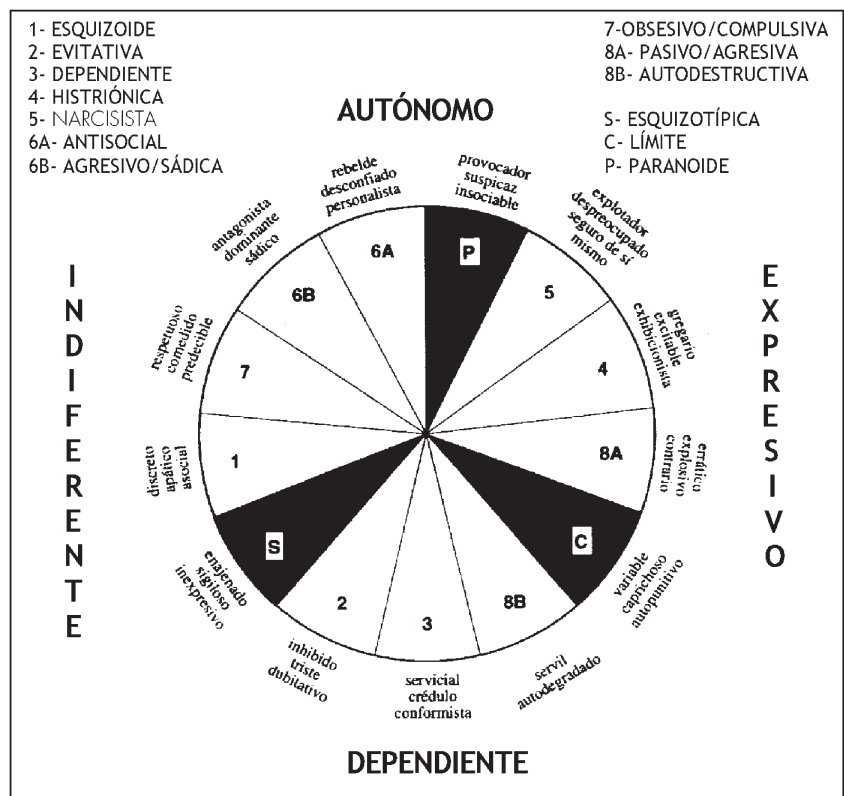

Gráfico 2. Modelo circumplejo de los trastornos de la personalidad del DSM-III-R (tomado del manual del MCMI-II).

\section{Procedimiento}

Se proporcionan ambos cuestionarios a los sujetos, instruyéndolos del mismo modo para la cumplimentación que se realiza en su propia casa, debiendo entregarse los tests en las siguientes 48 horas al profesional responsable del CAD. En todos los casos se advierte que esta evaluación forma parte de su proceso de tratamiento y que los datos podrán ser utilizados anónimamente en estudios de investigación si no se objeta al respecto por su parte.

\section{Análisis de datos}

Se ha utilizado el paquete estadístico SPSS 10.0 para Windows. Se han realizado estudios descriptivos de tendencia central, dispersión y rango de puntuaciones, correlación bivariada y análisis de regresión por pasos sucesivos.

\section{RESULTADOS}

Para los estudios posteriores se prescindió de los sujetos que emitieron datos distorsionados, eliminándose a aquellos que en la variable Distorsión del BFO puntuaban fuera de la $M \pm 1$ '5 desviaciones típicas; y aquéllos que puntuaban $>1$ en la escala $V$ y menos de 145 o más de 590 en la escala X del MCMI-II. La muestra quedó reducida a 166 sujetos.

En primer lugar, describimos las características generales de la muestra empleada. En la Tabla 1 se muestran las variables demográficas y otras relacionadas con el consumo de sustancias de la muestra general y de los grupos conformados en función de la sustancia que motiva la demanda de tratamiento.

TABLA 1. Variables demográficas y de consumo en la muestra general y en subgrupos según la droga que motiva la demanda de tratamiento

\begin{tabular}{|c|c|c|c|c|c|c|c|}
\hline & & Total & Alcohol & Cocaína & Heroína & Cannabis & Benzodiazepinas \\
\hline \multicolumn{2}{|l|}{$\mathbf{N}$} & 166 & 34 & 52 & 70 & 8 & 2 \\
\hline \multirow[t]{3}{*}{ Edad } & Media & 34,3 & 39.3 & 31,1 & 34.1 & 31,8 & 39,0 \\
\hline & DT & 7,4 & 8.4 & 6,3 & 5.6 & 7,8 & 9,9 \\
\hline & Rango & $19-63$ & $23-63$ & $20-47$ & $19-46$ & $23-45$ & $32-46$ \\
\hline \multirow[t]{2}{*}{ Sexo } & Varones & 121 & 23 & 44 & 49 & 5 & 0 \\
\hline & Mujeres & 45 & 11 & 8 & 21 & 3 & 2 \\
\hline \multirow{3}{*}{$\begin{array}{l}\text { Edad de inicio } \\
\text { en el consumo }\end{array}$} & Media & 19,5 & 19.4 & 20,2 & 19.2 & 15,9 & 26,0 \\
\hline & DT & 5,6 & 6,9 & 4,9 & 4,9 & 3,1 & 8,5 \\
\hline & Rango & $12-42$ & $12-38$ & $13-36$ & $13-42$ & $13-20$ & $20-32$ \\
\hline \multirow[t]{3}{*}{ Tiempo de consumo } & Media & 14,9 & 19,9 & 10,9 & 15.2 & 13,0 & 13,0 \\
\hline & DT & 7,4 & 7,8 & 6,3 & 6.0 & 6,5 & 1,4 \\
\hline & Rango & $1-42$ & $5-42$ & $1-30$ & $3-26$ & $7-22$ & $12-14$ \\
\hline
\end{tabular}


Primeramente describimos las puntuaciones de la muestra con relación a su personalidad normal. En la Tabla 2 se presentan los valores obtenidos en las dimensiones y subdimensiones del BFQ, tanto en puntuaciones directas como tipificadas:

TABLA 2. Descriptivos de la muestra total en sus puntuaciones en el BFQ ( $N=140)$

\begin{tabular}{|c|c|c|c|c|c|}
\hline & \multicolumn{4}{|c|}{$\begin{array}{l}\text { Puntuaciones } \\
\text { directas }\end{array}$} & \multirow{2}{*}{$\begin{array}{l}\text { Punt. } \\
\frac{\mathrm{T}}{\text { Media }}\end{array}$} \\
\hline & Media & D.T. & Mín. & Máx. & \\
\hline ENERGÍA & 76,0 & 9,0 & 48 & 98 & 50 \\
\hline Dinamismo & 39,1 & 6,0 & 23 & 53 & 49 \\
\hline Dominancia & 36,9 & 5,0 & 24 & 53 & 49 \\
\hline$A F A B I L I D A D$ & 80,7 & 9,1 & 61 & 110 & 49 \\
\hline Cooperatividad & 43,3 & 5,4 & 27 & 57 & 50 \\
\hline Cordialidad & 37,2 & 5,8 & 12 & 55 & 47 \\
\hline TESÓN & 76,8 & 10,7 & 45 & 111 & 47 \\
\hline Escrupulosidad & 37,3 & 6,4 & 23 & 55 & 47 \\
\hline Perseverancia & 39,5 & 6,5 & 22 & 59 & 46 \\
\hline ESTABILIDAD EMOCIONAL & 59,0 & 13,9 & 24 & 103 & 42 \\
\hline Control de las emociones & 29,0 & 7,8 & 13 & 55 & 42 \\
\hline Control de los impulsos & 29,2 & 7,4 & 13 & 51 & 43 \\
\hline APERTURA MENTAL & 78,7 & 12,3 & 40 & 110 & 46 \\
\hline Apertura a la cultura & 39,6 & 7,5 & 18 & 57 & 46 \\
\hline Apertura a la experiencia & 39,7 & 5,5 & 23 & 56 & 45 \\
\hline DISTORSIÓN & 26,9 & 5,3 & 19 & 38 & 49 \\
\hline
\end{tabular}

A continuación se estudiaron las puntuaciones en el BFQ, desglosando los grupos en función de la droga que motiva la demanda. Los resultados se presentan en la Tabla 3.
En la Tabla 4 se presentan las correlaciones encontradas entre las dimensiones del BFO en nuestra muestra y en la población normativa española (Caprara et al., 1995).

Abordamos a continuación la descripción de la muestra, en esta ocasión desde la perspectiva de la personalidad patológica, mediante los resultados obtenidos con el MCMI-II. En la Tabla 5 se muestran los valores medios, la dispersión y el rango de puntuaciones directas obtenidas por cada subgrupo -según la droga que motiva la demanda- y por la muestra total en cada patrón disfuncional.

Una vez realizada la transformación de las puntuaciones directas en puntuaciones de Tasa Base, en función de la prevalencia estimada en submuestras de adictos, se muestra en la Tabla 6 la distribución de frecuencias de las puntuaciones menores de 75 (que sugieren la ausencia de trastorno), entre 75 y 84 (sugestivas de presencia del trastorno) y superiores a 84 (presencia en grado de severidad del trastorno) según la droga que motiva la demanda de tratamiento.

En la Tabla 7 se presentan las correlaciones entre las escalas del MCMI-II de nuestra muestra y las que el autor propone en el manual del cuestionario en español (Millon, 1999).

Abordamos a continuación el objetivo del presente trabajo: establecer las relaciones entre las escalas de personalidad normal y las de los patrones desadaptativos estimados dimensionalmente. Para ello, se consideran las correlaciones entre las puntuaciones directas de las dimensiones y subdimensiones del BFO y de las escalas del MCMI-II (Tabla 8). Sin embar-

TABLA 3. Descriptivos de la muestra, desglosada por grupos según la droga que motiva la demanda de tratamiento, en sus puntuaciones en el BFQ $(\mathrm{N}=166)$.

\begin{tabular}{|c|c|c|c|c|c|c|c|c|c|c|}
\hline & \multicolumn{2}{|l|}{ Heroína } & \multicolumn{2}{|l|}{ Cocaina } & \multicolumn{2}{|l|}{ Alcohol } & \multicolumn{2}{|l|}{ Cannabis } & \multicolumn{2}{|c|}{ Benzodiazep. } \\
\hline & PD & $\mathbf{T}$ & PD & $\mathbf{T}$ & PD & $\mathbf{T}$ & PD & $\mathbf{T}$ & PD & $\mathbf{T}$ \\
\hline ENERGÍA & $75,5( \pm 8,5)$ & 49 & $77,7( \pm 8,4)$ & 51 & $74,2( \pm 10,0)$ & 47 & $76,9( \pm 9,8)$ & 50 & $73,0( \pm 19,8)$ & 46 \\
\hline Dinamismo & $38,9( \pm 5,9)$ & 47 & $39,7( \pm 5,8)$ & 49 & $38,3( \pm 6,6)$ & 47 & $41,1( \pm 4,8)$ & 53 & $40,5( \pm 9,2)$ & 50 \\
\hline Dominancia & $36,8( \pm 4,3)$ & 49 & $38,0( \pm 5,4)$ & 53 & $35,8( \pm 4,9)$ & 47 & $35,9( \pm 6,6)$ & 47 & $32,5( \pm 10,6)$ & 44 \\
\hline AFABILIDAD & $80,4( \pm 8,2)$ & 49 & $80,3( \pm 8,7)$ & 49 & $81,1( \pm 11,5)$ & 50 & $82,4( \pm 6,9)$ & 51 & $92,5( \pm 2,1)$ & 60 \\
\hline Cooperatividad & $42,9( \pm 4,9)$ & 49 & $43,5( \pm 5,7)$ & 50 & $42,6( \pm 5,8)$ & 49 & $45,6( \pm 5,1)$ & 56 & $50,0( \pm 1,4)$ & 63 \\
\hline Cordialidad & $37,1( \pm 5,5)$ & 47 & $37,1( \pm 5,5)$ & 47 & $37,9( \pm 6,8)$ & 47 & $36,9( \pm 4,4)$ & 44 & $42,5( \pm 0,7)$ & 54 \\
\hline TESÓN & $77,7( \pm 10,2)$ & 47 & $75,0 \quad( \pm 10,9)$ & 44 & $77,4( \pm 11,8)$ & 47 & $76,7( \pm 10,7)$ & 46 & $83,5( \pm 4,9)$ & 53 \\
\hline Escrupulosidad & $38,4( \pm 6,2)$ & 49 & $35,4( \pm 6,2)$ & 44 & $37,7( \pm 6,7)$ & 47 & $37,4( \pm 4,8)$ & 47 & $41,0( \pm 11,3)$ & 54 \\
\hline Perseverancia & $39,5( \pm 6,1)$ & 46 & $39,4( \pm 6,7)$ & 46 & $39,6( \pm 7,3)$ & 46 & $39,2( \pm 7,0)$ & 46 & $42,5( \pm 6,4)$ & 49 \\
\hline ESTABILIDAD EMOCIONAL & $59,8( \pm 12,5)$ & 42 & $57,6( \pm 13,9)$ & 41 & $57,2( \pm 15,9)$ & 41 & $72,5( \pm 8,5)$ & 52 & $44,0( \pm 12,7)$ & 34 \\
\hline Control de las Emociones & $30,5( \pm 6,9)$ & 42 & $29,0( \pm 7,3)$ & 42 & $28,9( \pm 9,0)$ & 40 & $37,0( \pm 9,4)$ & 53 & $19,0( \pm 1,4)$ & 32 \\
\hline Control de los impulsos & $29,1( \pm 7,0)$ & 43 & $29,0( \pm 7,5)$ & 43 & $28,5( \pm 8,1)$ & 42 & $35,7( \pm 3,4)$ & 53 & $25,0( \pm 1,3)$ & 40 \\
\hline APERTURA MENTAL & $78,3( \pm 12,5)$ & 44 & $77,2( \pm 11,7)$ & 44 & $80,8( \pm 13,1)$ & 47 & $83,6( \pm 10,9)$ & 50 & $82,0( \pm 9,9)$ & 47 \\
\hline Apertura a la cultura & $39,4( \pm 7,5)$ & 46 & $38,2( \pm 7,3)$ & 44 & $41,2( \pm 7,7)$ & 49 & $43,0( \pm 6,4)$ & 53 & $43,5( \pm 3,5)$ & 53 \\
\hline Apertura a la experiencia & $39,5( \pm 5,5)$ & 44 & $39,4( \pm 5,6)$ & 44 & $40,2( \pm 5,3)$ & 47 & $40,6( \pm 4,7)$ & 47 & $38,5( \pm 13,4)$ & 43 \\
\hline DISTORSIÓN & $26,3( \pm 6,5)$ & 47 & $26,1( \pm 4,9)$ & 47 & $28,2( \pm 5,4)$ & 50 & $31,7( \pm 12,3)$ & 54 & $24,5( \pm 6,4)$ & 43 \\
\hline ntuaciones Directas en el BFQ & $T=$ Pur & & n el BFQ & & & & & & & \\
\hline
\end{tabular}


Tabla 4. Correlaciones apreciadas entre las dimensiones del BFQ en nuestra muestra (sobre la diagonal) y en la muestra normativa de población española (bajo la diagonal).

\begin{tabular}{|c|c|c|c|c|c|}
\hline & ENERGÍA & AFABILIDAD & TESÓN & ESTABILIDAD EMOCIONAL & APERTURA MENTAL \\
\hline ENERGÍA & &, $36(* *)$ &, $32(* *)$ &,- 10 &, $41(* *)$ \\
\hline AFABILIDAD &, 21 & &, $38\left({ }^{* *}\right)$ &, 14 &, $41\left(*^{*}\right)$ \\
\hline TESÓN &, 35 &, 15 & &, $16\left(^{*}\right)$ &, $35(* *)$ \\
\hline ESTABILIDAD EMOCIONAL &,- 04 &, 09 &, 04 & &, 04 \\
\hline APERTURA MENTAL & ,39 & ,39 &, 31 &, 01 & \\
\hline
\end{tabular}

Tabla 5. Descriptivos de la muestra, desglosada por grupos según la droga que motiva la demanda de tratamiento, en sus puntuaciones directas en el MCMI-II ( $\mathrm{N}=166)$

\begin{tabular}{|c|c|c|c|c|c|c|c|c|c|c|c|}
\hline & & $\mathbf{M}$ & D.T. & Mín & Máx & & & $\mathbf{M}$ & D.T. & Mín & Máx \\
\hline \multirow{6}{*}{ ESQUIZOIDE } & Heroina & 23,3 & 6,6 & 9 & 40 & \multirow{6}{*}{ COMPULSIVA } & Heroina & 35,9 & 8,0 & 14 & 48 \\
\hline & Cocaina & 23,1 & 7,9 & 10 & 50 & & Cocaina & 33,0 & 7,3 & 19 & 48 \\
\hline & Alcohol & 22,6 & 7,7 & 4 & 43 & & Alcohol & 35,9 & 7,8 & 21 & 55 \\
\hline & Cannabis & 16,2 & 4,7 & 9 & 21 & & Cannabis & 35,2 & 9,8 & 14 & 47 \\
\hline & Benzodiaz. & 25,5 & 13,4 & 16 & 35 & & Benzodiaz. & 33,0 & 12,7 & 24 & 42 \\
\hline & Total & 22,8 & 7,3 & 4 & 50 & & Total & 35,0 & 7,9 & 14 & 55 \\
\hline \multirow{6}{*}{ FÓBICA } & Heroina & 27,3 & 12,2 & 4 & 60 & \multirow{6}{*}{ PASIVOAGRESIVA } & Heroina & 36,7 & 12,7 & 10 & 68 \\
\hline & Cocaina & 28,3 & 11,5 & 5 & 58 & & Cocaina & 40,5 & 14,1 & 13 & 64 \\
\hline & Alcohol & 28,9 & 12,0 & 3 & 55 & & Alcohol & 38,1 & 14,9 & 8 & 70 \\
\hline & Cannabis & 15,6 & 8,4 & 2 & 27 & & Cannabis & 24,3 & 12,7 & 5 & 42 \\
\hline & Benzodiaz. & 36,0 & 9,9 & 29 & 43 & & Benzodiaz. & 42,0 & 12,7 & 33 & 51 \\
\hline & Total & 27,5 & 12,0 & 2 & 60 & & Total & 37,7 & 13,9 & 5 & 70 \\
\hline \multirow{6}{*}{ DEPENDIENTE } & Heroina & 30,2 & 8,0 & 11 & 44 & \multirow{6}{*}{ AUTODESTRUCTIVA } & Heroina & 28,3 & 12,7 & 4 & 57 \\
\hline & Cocaina & 31,5 & 7,7 & 14 & 45 & & Cocaina & 30,0 & 13,4 & 7 & 58 \\
\hline & Alcohol & 31,5 & 8,5 & 9 & 45 & & Alcohol & 28,8 & 10,6 & 2 & 46 \\
\hline & Cannabis & 27,7 & 6,7 & 17 & 36 & & Cannabis & 12,6 & 10,0 & 2 & 28 \\
\hline & Benzodiaz. & 27,5 & 3,5 & 25 & 30 & & Benzodiaz. & 43,5 & 5,0 & 40 & 47 \\
\hline & Total & 30,7 & 7,9 & 9 & 45 & & Total & 28,4 & 12,9 & 2 & 58 \\
\hline \multirow{6}{*}{ HISTRIÓNICA } & Heroina & 34,2 & 9,6 & 12 & 54 & \multirow{6}{*}{ ESQUIZOTÍPICA } & Heroina & 23,1 & 11,6 & 5 & 52 \\
\hline & Cocaina & 36,8 & 10,1 & 20 & 58 & & Cocaina & 24,2 & 12,8 & 1 & 58 \\
\hline & Alcohol & 36,4 & 11,4 & 10 & 56 & & Alcohol & 25,0 & 9,8 & 8 & 44 \\
\hline & Cannabis & 27,1 & 9,1 & 19 & 46 & & Cannabis & 13,3 & 7,2 & 5 & 24 \\
\hline & Benzodiaz. & 26,0 & 12,7 & 17 & 35 & & Benzodiaz. & 27,5 & 10,6 & 20 & 35 \\
\hline & Total & 35,0 & 10,3 & 10 & 58 & & Total & 23,4 & 11,6 & 1 & 58 \\
\hline \multirow{6}{*}{ NARCISISTA } & Heroina & 39,8 & 9,9 & 17 & 62 & \multirow{6}{*}{ LÍMITE } & Heroina & 47,1 & 18,1 & 10 & 86 \\
\hline & Cocaina & 39,8 & 9,5 & 18 & 61 & & Cocaina & 50,0 & 20,4 & 12 & 86 \\
\hline & Alcohol & 40,7 & 13,2 & 12 & 67 & & Alcohol & 48,2 & 17,8 & 9 & 80 \\
\hline & Cannabis & 35,9 & 9,0 & 25 & 49 & & Cannabis & 23,4 & 14,2 & 2 & 41 \\
\hline & Benzodiaz. & 34,5 & 10,6 & 27 & 42 & & Benzodiaz. & 70,0 & 12,7 & 61 & 79 \\
\hline & Total & 39,7 & 10,5 & 12 & 67 & & Total & 47,3 & 19,4 & 2 & 86 \\
\hline \multirow{6}{*}{ ANTISOCIAL } & Heroina & 40,5 & 10,7 & 16 & 71 & \multirow{6}{*}{ PARAN } & Heroina & 32,3 & 11,1 & 6 & 62 \\
\hline & Cocaina & 40,5 & 12,1 & 16 & 63 & & Cocaina & 32,3 & 11,0 & 11 & 52 \\
\hline & Alcohol & 40,6 & 13,4 & 18 & 63 & & Alcohol & 32,9 & 10,4 & 10 & 51 \\
\hline & Cannabis & 31,6 & 9,6 & 15 & 45 & & Cannabis & 27,0 & 10,4 & 14 & 44 \\
\hline & Benzodiaz. & 36,5 & 19,1 & 23 & 50 & & Benzodiaz. & 26,5 & 2,1 & 25 & 28 \\
\hline & Total & 40,1 & 11,8 & 15 & 71 & & Total & 32,1 & 10,8 & 6 & 62 \\
\hline \multirow{6}{*}{ AGRESIVO SÁDICA } & Heroina & 37,3 & 10,8 & 11 & 63 & & & & & & \\
\hline & Cocaina & 37,7 & 10,9 & 16 & 58 & & & & & & \\
\hline & Alcohol & 37,3 & 14,8 & 13 & 63 & & & & & & \\
\hline & Cannabis & 29,8 & 10,1 & 15 & 45 & & & & & & \\
\hline & Benzodiaz. & 28,0 & 8,5 & 22 & 34 & & & & & & \\
\hline & Total & 36,9 & 11,7 & 11 & 63 & & & & & & \\
\hline
\end{tabular}


Tabla 6. Frecuencia de puntuaciones sugestivas de ausencia (TB < 75), presencia $(74<$ TB $<85$ ) y severidad (TB > 84) de cada trastorno, según las puntuaciones de Tasa Base en cada subgrupo según la droga que motiva la demanda.

\begin{tabular}{|c|c|c|c|c|c|c|c|c|c|c|c|c|c|c|c|}
\hline & \multicolumn{5}{|c|}{ TB $<75$} & \multicolumn{5}{|c|}{$74<$ TB $<85$} & \multicolumn{5}{|c|}{ TB $>84$} \\
\hline & $\mathrm{H}$ & C & A & $\mathrm{CN}$ & BZ & $\mathrm{H}$ & C & A & $\mathrm{CN}$ & $B Z$ & $\mathrm{H}$ & C & A & $\mathrm{CN}$ & $B Z$ \\
\hline ESOUIZOIDE & 64 & 48 & 31 & 8 & 1 & 3 & 0 & 1 & 0 & 0 & 3 & 4 & 2 & 0 & 1 \\
\hline FÓBICA & 58 & 44 & 24 & 8 & 1 & 3 & 5 & 6 & 0 & 0 & 9 & 3 & 4 & 0 & 1 \\
\hline DEPENDIENTE & 56 & 38 & 23 & 8 & 2 & 10 & 12 & 5 & 0 & 0 & 4 & 2 & 6 & 0 & 0 \\
\hline HISTRIÓNICA & 53 & 37 & 22 & 7 & 2 & 12 & 8 & 8 & 1 & 0 & 5 & 7 & 4 & 0 & 0 \\
\hline NARCISISTA & 53 & 38 & 23 & 7 & 2 & 11 & 7 & 3 & 0 & 0 & 6 & 7 & 8 & 1 & 0 \\
\hline ANTISOCIAL & 53 & 34 & 22 & 8 & 1 & 8 & 11 & 3 & 0 & 0 & 9 & 7 & 9 & 0 & 1 \\
\hline AGRESIVO SÁDICA & 50 & 32 & 20 & 6 & 2 & 8 & 9 & 4 & 2 & 0 & 12 & 11 & 10 & 0 & 0 \\
\hline COMPULSIVA & 60 & 50 & 30 & 6 & 2 & 9 & 2 & 3 & 1 & 0 & 1 & 0 & 1 & 1 & 0 \\
\hline PASIVO AGRESIVA & 51 & 30 & 25 & 8 & 1 & 12 & 4 & 2 & 0 & 1 & 7 & 18 & 7 & 0 & 0 \\
\hline AUTODESTRUCTIVA & 55 & 38 & 29 & 8 & 0 & 8 & 5 & 4 & 0 & 1 & 7 & 9 & 1 & 0 & 1 \\
\hline ESQUIZOTÍPICA & 61 & 48 & 32 & 8 & 2 & 8 & 2 & 2 & 0 & 0 & 1 & 2 & 0 & 0 & 0 \\
\hline LÍMITE & 56 & 42 & 29 & 8 & 1 & 5 & 7 & 0 & 0 & 0 & 9 & 3 & 5 & 0 & 1 \\
\hline PARANOIDE & 65 & 47 & 31 & 7 & 2 & 1 & 5 & 3 & 1 & 0 & 4 & 0 & 0 & 0 & 0 \\
\hline
\end{tabular}

$\mathrm{H}=$ Heroína; $\mathrm{C}=$ Cocaína; $\mathrm{A}=$ Alcohol; $\mathrm{CN}=$ Cannabis; $\mathrm{BZ}=$ Benzodiazepinas.

Tabla 7. Correlaciones apreciadas entre las escalas del MCMI-II en nuestra muestra (sobre la diagonal) y en la muestra normativa ofrecida en el manual del cuestionario (bajo la diagonal).

\begin{tabular}{|c|c|c|c|c|c|c|c|c|c|c|c|c|c|}
\hline & 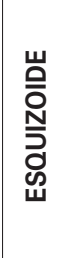 & 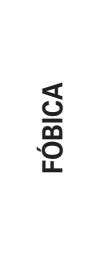 & $\begin{array}{l}\text { 岁 } \\
\text { 訔 } \\
\text { 岀 } \\
\text { 㟔 }\end{array}$ & 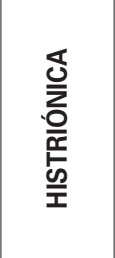 & 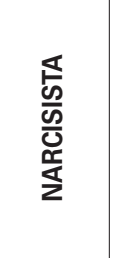 & 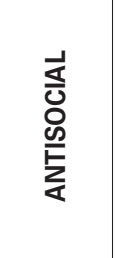 & 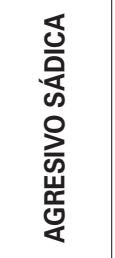 & 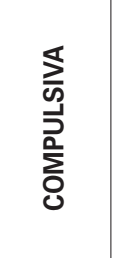 & 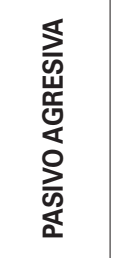 & 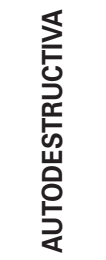 & 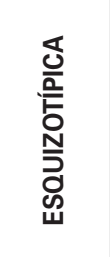 & 莺 & 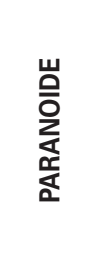 \\
\hline ESQUIZOIDE & &, $69\left({ }^{* *}\right)$ &, $19\left({ }^{*}\right)$ &,$- 37\left({ }^{* *}\right)$ &,- 13 & 14 &, 12 &, 11 &, $24\left({ }^{* *}\right)$ &, $46\left({ }^{* *}\right)$ &, $69(* *)$ &, $40(* *)$ &, $22\left({ }^{* *}\right)$ \\
\hline FÓBICA & 62 & &, $33(* *)$ &,- 01 &,- 03 &, $32(* *)$ &, $26(* *)$ &,- 11 &, $56(* *)$ &, $83(* *)$ &, $92(* *)$ &, $75(* *)$ &, $36(* *)$ \\
\hline DEPENDIENTE & 27 & ,34 & &, 12 &,$- 22\left({ }^{* *}\right)$ &,$- 29(* *)$ &,$- 30(* *)$ & $22\left({ }^{* *}\right)$ & 01 &, $46(* *)$ &, $30(* *)$ &, $20\left(^{*}\right)$ &, 08 \\
\hline HISTRIÓNICA &,- 59 &,- 29 &,- 11 & &, $72\left({ }^{* *}\right)$ &, $55(* *)$ &, $52(* *)$ &,$- 19\left(^{*}\right)$ &, $54\left({ }^{* *}\right)$ &, $28\left({ }^{* *}\right)$ & 01 &, $41(* *)$ &, $44\left({ }^{* *}\right)$ \\
\hline NARCISISTA &,- 35 &,- 22 &,- 41 & ,69 & &, $67\left({ }^{* *}\right)$ &, $73\left({ }^{* *}\right)$ &, 00 &, $52\left({ }^{* *}\right)$ &, 11 &, 05 &, $31(* *)$ &, $68\left(^{* *}\right)$ \\
\hline ANTISOCIAL &,- 12 & 18 &,- 34 & ,48 & 65 & &, $76\left({ }^{* *}\right)$ &,$- 23\left({ }^{* *}\right)$ &, $73\left({ }^{* *}\right)$ &, $34\left({ }^{* *}\right)$ &, $40\left({ }^{* *}\right)$ &, $61(* *)$ &, $62\left({ }^{* *}\right)$ \\
\hline AGRESIVO SÁDICA &,- 13 & ,02 &,- 46 & ,43 & ,71 & ,73 & &, 10 &, $72\left({ }^{* *}\right)$ &, $30\left({ }^{* *}\right)$ &, $30(* *)$ &, $51\left({ }^{* *}\right)$ &, $73\left({ }^{* *}\right)$ \\
\hline COMPULSIVA & ,23 &,- 09 &, 16 &,- 29 &,- 05 &,- 27 & 01 & &,$- 24\left({ }^{* *}\right)$ &,- 13 &,- 06 &,$- 24\left({ }^{* *}\right)$ &, $33\left({ }^{* *}\right)$ \\
\hline PASIVO AGRESIVA & 15 &, 58 &,- 04 & ,26 & ,34 & ,66 & ,58 &,- 30 & &, $69(* *)$ &, $58(* *)$ &, $84(* *)$ &, $62(* *)$ \\
\hline AUTODESTRUCTIVA & ,38 & 78 & ,47 &,- 03 &,- 12 & ,22 & ,05 &,- 19 & ,66 & &, $74(* *)$ &, $88(* *)$ &, $38\left(^{* *}\right)$ \\
\hline ESQUIZOTÍPICA &, 56 & ,76 & ,27 &,- 22 &,- 06 & ,27 & 14 & ,05 &, 53 & ,65 & &, $71(* *)$ &, $47(* *)$ \\
\hline LÍMITE & 17 & 62 & ,14 & ,25 & ,22 &, 56 & ,42 &,- 23 & ,79 & ,76 &, 56 & &, $46\left({ }^{* *}\right)$ \\
\hline PARANOIDE & ,04 & ,23 &,- 07 & ,35 & ,60 &, 59 & ,63 & 24 &, 52 & ,25 & ,38 & ,47 & \\
\hline
\end{tabular}

go, se hace preciso también considerar las correlaciones con las puntuaciones de tasa base, en la medida en que para su obtención es preciso realizar una serie de correcciones sobre las puntuaciones directas que pueden alterar las anteriores. Para ello, y tras la transformación realizada siguiendo las sucesivas instrucciones del autor, en la Tabla 9 se muestran las correlaciones entre las puntuaciones tipificadas del BFQ y las puntuaciones TB del MCMI-II.

En la Tabla 10 constatamos los coeficientes Beta estandarizados obtenidos al realizar un análisis de regresión por pasos sucesivos de las dimensiones y subdimensiones del BFQ (introducidas por separado) sobre cada una de las escalas de patrones disfuncionales del MCMI-II, constatando únicamente aquéllas que han resultado significativas ( $F=0,05$ y 0,10 , de entrada y salida, respectivamente).

Finalmente, en la Tabla 11 se representan gráficamente las concordancias entre ambos métodos (correlación bivariada y análisis de regresión lineal) en la estimación de relaciones entre las dimensiones y subdimensiones del BFO y las escalas del MCMI-II. 
Tabla 8. Correlaciones entre las dimensiones y subdimensiones del BFO y las escalas del MCMI-2, a partir de las puntuaciones directas de ambos cuestionarios.

\begin{tabular}{|c|c|c|c|c|c|c|c|c|c|c|c|c|c|}
\hline & 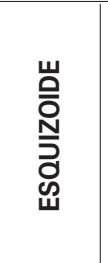 & 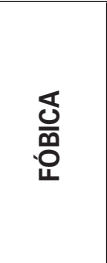 & $\begin{array}{l}\text { 岁 } \\
\text { 訔 } \\
\text { 岀 } \\
\text { 岀 }\end{array}$ & 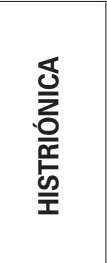 & 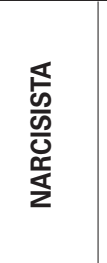 & 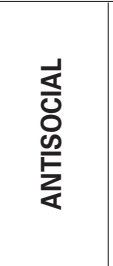 & 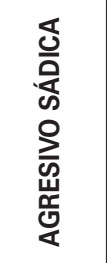 & 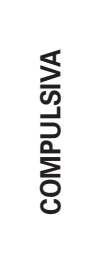 & 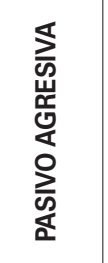 & 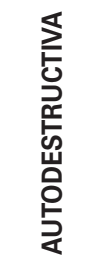 & 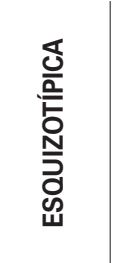 & $\stackrel{\underline{\underline{E}}}{\stackrel{\underline{\underline{E}}}{\Xi}}$ & 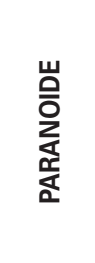 \\
\hline ENERGIA &,$- 40(* *)$ & $-29(* *)$ &,- 05 &, $45(* *)$ &, $41(* *)$ & ,13 &, $22(* *)$ &, 07 &, 08 &,- 10 & $-25(* *)$ &,- 05 &, $20(* *)$ \\
\hline Dinamismo &,$- 45(* *)$ & $-35(* *)$ &, 04 &, $35(* *)$ &, $23(* *)$ &,- 03 &,- 01 &, 05 &,- 06 &,- 13 & $-31(* *)$ &,- 13 &, 02 \\
\hline Dominancia &,$- 19(*)$ &,- 11 &,- 13 &, $40(* *)$ &, $47(* *)$ &, $29(* *)$ &, $42(* *)$ &, 08 &, $21(* *)$ &,- 01 &,- 07 &, 07 &, $35(* *)$ \\
\hline AFABILIDAD & $-36(* *)$ & $-24(* *)$ &, $32(* *)$ &, 11 &,$- 16\left(^{*}\right)$ & $-34(* *)$ & $-35(* *)$ & .05 & $-24(* *)$ &,- 03 & $-30(* *)$ &,$- 16\left(^{*}\right)$ &,$- 25(* *)$ \\
\hline Cooperatividad &,$- 35(* *)$ & $-24(* *)$ &, $17\left(^{*}\right)$ & ,11 &,- 03 & $-24(* *)$ & $-23(* *)$ &, 04 &,$- 18\left(^{*}\right)$ &,- 07 & $-28(* *)$ &,$- 18\left(^{*}\right)$ &,- 13 \\
\hline Cordialidad &,$- 19\left(^{*}\right)$ &,- 09 &, $34(* *)$ &, 08 &,$- 15\left(^{*}\right)$ & $-27(* *)$ & $-28(* *)$ &, 07 &,- 15 &, 04 &,$- 15\left(^{*}\right)$ &,- 09 &,$- 18\left({ }^{*}\right)$ \\
\hline TESÓN & $-21(* *)$ &,$- 25(* *)$ &, 06 &,- 04 &, 02 &,$- 18\left(^{*}\right)$ &,- 06 &, $43(* *)$ & $-25(* *)$ &,$- 23(* *)$ & $-24(* *)$ & $-29(* *)$ &, 11 \\
\hline Escrupulosidad &,- 06 &,- 10 &, 13 &,- 07 &,- 02 & $-21(* *)$ &,- 06 &, $46(* *)$ & $-20(* *)$ &,- 09 &,- 10 &,$- 18\left(^{*}\right)$ &, 10 \\
\hline Perseverancia & $-30(* *)$ & $-32(* *)$ &,- 07 &, 01 &, 08 &,- 08 &, 01 &, $31(* *)$ & $-21(* *)$ &,$- 30(* *)$ & $-32(* *)$ & $-31(* *)$ &, 11 \\
\hline ESTAB. EMOCIONAL &,- 03 & $-31(* *)$ &, 12 &,$- 33(* *)$ &,$- 26(* *)$ &,$- 40(* *)$ & $-36(* *)$ &, $27(* *)$ & $-58(* *)$ &,$- 47(* *)$ & $-28(* *)$ &,$- 56(* *)$ &,$- 22(* *)$ \\
\hline Control emociones &,- 10 &,$- 42(* *)$ &,- 04 & $-25(* *)$ &,- 12 & $-29(* *)$ & $-21(* *)$ &, $27(* *)$ &,$- 51(* *)$ &,$- 55(* *)$ & $-36(* *)$ &,$- 58(* *)$ &,- 14 \\
\hline Control impulsos &, 030 &,$- 16\left(^{*}\right)$ &, $23(* *)$ & $-34(* *)$ &,$- 32(* *)$ &,$- 44(* *)$ &,$- 43(* *)$ &, $22(* *)$ &,$- 53(* *)$ &,$- 31(* *)$ &,- 15 &,$- 43(* *)$ &,$- 26(* *)$ \\
\hline APERTURA MENTAL & $-36(* *)$ &,$- 32(* *)$ &,- 10 &, 13 &, 14 &,- 05 &,- 12 &, 04 & $-21(* *)$ &,$- 27(* *)$ & $-30(* *)$ &,$- 21(* *)$ &,- 07 \\
\hline Apert. a la cultura & $-29(* *)$ & $-31(* *)$ &,- 09 &, 03 &, 05 &,$- 18\left(^{*}\right)$ &,$- 16\left(^{*}\right)$ &, 11 & $-27(* *)$ &,$- 26(* *)$ &,$- 32(* *)$ & $-24(* *)$ &,$- 17\left(^{*}\right)$ \\
\hline Apert. a la experiencia & $-36(* *)$ & $-28(* *)$ &,- 10 &, $19\left(^{*}\right)$ & $21(* *)$ &, 06 &,- 08 &,- 08 &,- 12 &,$- 24(* *)$ & $-24(* *)$ &,- 15 &, 02 \\
\hline DISTORSIÓN &, 10 &,- 07 &, $34(* *)$ &, 05 &, 08 & $-20(* *)$ &,- 09 &, $37(* *)$ &,$- 15(*)$ &,- 01 &,- 01 &,- 13 &, 15 (*) $^{*}$ \\
\hline
\end{tabular}

Tabla 9. Correlaciones entre las dimensiones y subdimensiones del BFO y las escalas del MCMI-2, a partir de las puntuaciones Tipificadas del BFO y de las puntuaciones TB (de tasa base) del MCMI-II.

\begin{tabular}{|c|c|c|c|c|c|c|c|c|c|c|c|c|c|}
\hline & $\begin{array}{l}\text { 山् } \\
\text { 옹 } \\
\text { 었 }\end{array}$ & $\begin{array}{l}\text { త్ } \\
\text { 。्ञㅁ }\end{array}$ & 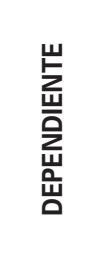 & 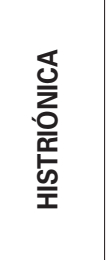 & 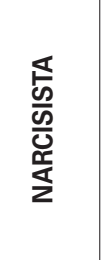 & 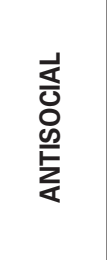 & 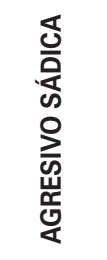 & 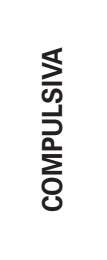 & 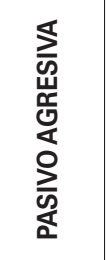 & 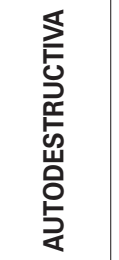 & $\begin{array}{l}\text { 음 } \\
\text { 음 } \\
\text { N } \\
\text { 였 }\end{array}$ & $\underset{\underline{\underline{E}}}{\stackrel{\underline{\underline{E}}}{\boldsymbol{E}}}$ & 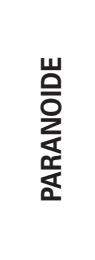 \\
\hline ENERGIA & $-42(* *)$ &,$- 31(* *)$ &,- 05 &, $51(* *)$ &, $47(* *)$ &, 13 &, $25(* *)$ & ,09 & , 10 &,- 12 &,$- 23(* *)$ &,- 09 &, $30(* *)$ \\
\hline Dinamismo &,$- 45(* *)$ &,$- 34(* *)$ &, 06 &, $42(* *)$ &, $32(* *)$ &,- 01 &, 03 &, 07 &,- 03 &,- 14 & $-30(* *)$ &,- 12 &, 13 \\
\hline Dominancia & $-24(* *)$ &,$- 16\left(^{*}\right)$ &,- 14 &, $44(* *)$ &, $48(* *)$ &, $25(* *)$ &, $41(* *)$ & ,08 &, $22(* *)$ &,- 05 &,- 09 &,- 02 &, $41(* *)$ \\
\hline AFABILIDAD & $-32(* *)$ &,$- 21(* *)$ &, $37(* *)$ &, $16\left(^{*}\right)$ &,- 08 & $-31(* *)$ & $-32(* *)$ &, 09 &,$- 22(* *)$ &, 01 & $-27(* *)$ &,- 04 &,- 09 \\
\hline Cooperatividad & $-32(* *)$ &,$- 20(* *)$ &, $22(* *)$ &, $16\left(^{*}\right)$ &, 03 & $-21(* *)$ &,$- 18\left(^{*}\right)$ &, 07 &,- 15 &,- 04 &,$- 24(* *)$ &,- 07 &, 03 \\
\hline Cordialidad &,$- 19(*)$ &,- 10 &, $39(* *)$ &, 09 &,- 12 & $-29(* *)$ &,$- 31(* *)$ &, 11 &,$- 18(*)$ &, 05 &,- 15 &,- 02 &,- 09 \\
\hline TESÓN &,$- 18\left(^{*}\right)$ &,$- 21(* *)$ & ,14 &, 01 &, 09 &,$- 16\left(^{*}\right)$ &,- 02 &, $43(* *)$ &,$- 21(* *)$ & $-20\left(^{*}\right)$ &,$- 21(* *)$ & $-27(* *)$ &, $26(* *)$ \\
\hline Escrupulosidad &,- 02 &,- 08 &, $17\left(^{*}\right)$ &,- 07 &, 02 &,$- 19(*)$ &,- 04 & , 44 (**) &,$- 19(*)$ &,- 06 &,- 10 &,- 15 &, $22(* *)$ \\
\hline Perseverancia &,$- 29(* *)$ &,$- 26(* *)$ &, 03 &, 09 &, 10 &,- 07 &, 07 &, $28(* *)$ &,- 15 &,$- 25(* *)$ & $-23(* *)$ & $-29(* *)$ &, $21(* *)$ \\
\hline ESTAB. EMOCIONAL &,- 01 &,$- 33(* *)$ &, $19\left(^{*}\right)$ & $-25(* *)$ &,$- 16(*)$ &,$- 40(* *)$ & $-31(* *)$ &, $33(* *)$ & $-59(* *)$ &,$- 48(* *)$ & $-26(* *)$ & $-43(* *)$ &,- 12 \\
\hline Control emociones &,- 08 &,$- 42(* *)$ &, 05 &,$- 16\left(^{*}\right)$ &,- 03 &,$- 27(* *)$ &,- 15 &, $32(* *)$ &,$- 48(* *)$ &,$- 56(* *)$ &,$- 33(* *)$ &,$- 50(* *)$ &,- 04 \\
\hline Control impulsos &, 05 &,- 14 &, $30(* *)$ & $-27(* *)$ & $-24(* *)$ &,$- 42(* *)$ &,$- 37(* *)$ &, $27(* *)$ &,$- 54(* *)$ & $-29(* *)$ &,- 11 & $-26(* *)$ &,- 15 \\
\hline APERTURA MENTAL & $-34(* *)$ &,$- 30(* *)$ &,- 03 &, 15 &, $18\left(^{*}\right)$ &,- 07 &,- 07 &, 07 &,$- 26(* *)$ & $-29(* *)$ &,$- 27(* *)$ &,$- 19\left(^{*}\right)$ &, 01 \\
\hline Apert. a la cultura &,$- 25(* *)$ &,$- 26(* *)$ &,- 02 &, 06 &, 11 &,$- 15(*)$ &,- 06 &, 13 &,$- 27(* *)$ &,$- 23(* *)$ &,$- 26(* *)$ &,- 14 &,- 03 \\
\hline Apert. a la experiencia &,$- 34(* *)$ &,$- 25(* *)$ &,- 06 &, $26(* *)$ &, $24(* *)$ &, 08 &,- 05 &,- 06 &,- 11 &,$- 24(* *)$ &,$- 21(* *)$ &,- 14 &, 06 \\
\hline DISTORSIÓN &,- 01 &,- 02 &, $37(* *)$ &, 08 &, 08 &,$- 22(* *)$ &,- 07 &, $34(* *)$ &,$- 15(*)$ &, 03 &, 07 &,- 02 &, $24(* *)$ \\
\hline
\end{tabular}


Tabla 10. Coeficientes beta estandarizados del análisis de regresión por pasos sucesivos de las dimensiones y subdimensiones (introducidas por separado) del BFQ sobre cada uno de las escalas del MCMI-II.

\begin{tabular}{|c|c|c|c|c|c|c|c|c|c|c|c|c|c|}
\hline & 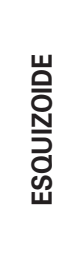 & $\begin{array}{l}\mathbb{J} \\
\frac{0}{0} \\
\text { 요 }\end{array}$ & $\begin{array}{l}\text { 世 } \\
\text { 岕 } \\
\text { 号 } \\
\text { 岃 } \\
\text { 岁 }\end{array}$ & 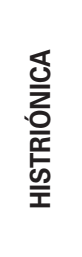 & 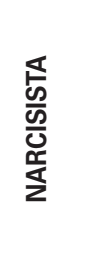 & 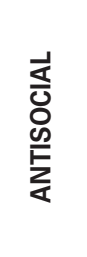 & 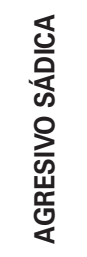 & 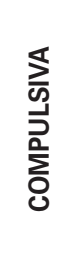 & 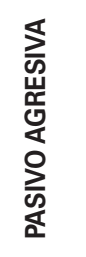 & 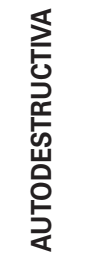 & 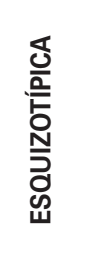 & 亗 & 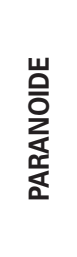 \\
\hline ENERGIA & $-0,34$ & $-0,26$ & $-0,16$ & 0,50 & 0,58 & 0,23 & 0,39 & & 0,17 & & $-0,18$ & & 0,33 \\
\hline Dinamismo & $-0,38$ & $-0,33$ & & 0,33 & & & & & & & $-0,29$ & & \\
\hline Dominancia & & & & 0,31 & 0,42 & 0,21 & 0,38 & & 0,19 & & & & 0,41 \\
\hline AFABILIDAD & & & 0,52 & & $-0,30$ & $-0,34$ & $-0,43$ & & & 0,23 & & & $-0,31$ \\
\hline Cooperatividad & & & & & & & & & & & & & \\
\hline Cordialidad & & & 0,38 & & & $-0,21$ & $-0,24$ & & & 0,13 & & & \\
\hline TESÓN & & & & & & & & 0,39 & & & & $-0,20$ & 0,27 \\
\hline Escrupulosidad & & & & $-0,15$ & & & & 0,45 & & & & & 0,22 \\
\hline Perseverancia & & & & & & & & & & & & $-0,20$ & \\
\hline ESTAB. EMOCIONAL & & $-0,33$ & & $-0,22$ & & $-0,32$ & $-0,21$ & 0,26 & $-0,55$ & $-0,48$ & $-0,25$ & $-0,40$ & \\
\hline Control emociones & & & & & & & & 0,28 & $-0,26$ & $-0,57$ & $-0,32$ & $-0,46$ & \\
\hline Control impulsos & & $-0,41$ & 0,23 & $-0,20$ & $-0,19$ & $-0,33$ & $-0,26$ & & $-0,33$ & & & & \\
\hline APERTURA MENTAL & $-0,20$ & $-0,16$ & $-0,19$ & & & & & & $-0,26$ & $-0,34$ & $-0,17$ & & \\
\hline Apert. a la cultura & & & $-0,18$ & & & & & & $-0,21$ & & & & $-0,16$ \\
\hline Apert. a la experiencia & $-0,17$ & & & & 0,16 & & & $-0,14$ & & $-0,27$ & & & \\
\hline
\end{tabular}

TABLA 11. Representación gráfica de las concordancia entre los métodos de correlación bivariada y el análisis de regresión por pasos sucesivos entre las dimensiones y subdimensiones del BFO (variables predictoras) y las escalas del MCMI-II (variable dependiente).

\begin{tabular}{|c|c|c|c|c|c|c|c|c|c|c|c|c|c|}
\hline & 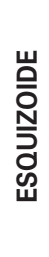 & 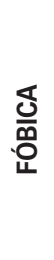 & 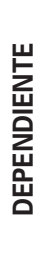 & 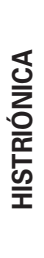 & 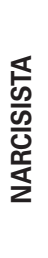 & 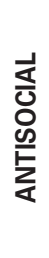 & 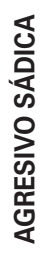 & 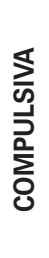 & 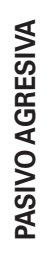 & 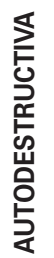 & 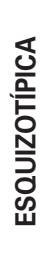 & 点 & 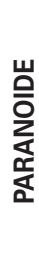 \\
\hline ENERGIA & - & - & & + & + & & + & & & & - & & \\
\hline Dinamismo & - & - & & + & & & & & & & - & & \\
\hline Dominancia & & & & + & + & + & + & & + & & & & + \\
\hline AFABILIDAD & & & + & & & - & - & & & & & & - \\
\hline Cooperatividad & & & & & & & & & & & & & \\
\hline Cordialidad & & & + & & & - & - & & & & & & \\
\hline TESÓN & & & & & & & & + & & & & - & \\
\hline Escrupulosidad & & & & & & & & + & & & & & \\
\hline Perseverancia & & & & & & & & & & & & - & \\
\hline ESTAB. EMOCIONAL & & - & & - & & - & - & + & - & - & - & - & \\
\hline Control emociones & & & & & & & & + & - & - & - & - & \\
\hline Control impulsos & & - & + & - & - & - & - & & - & & & & \\
\hline APERTURA MENTAL & - & - & & & & & & & - & - & - & & \\
\hline Apert. a la cultura & & & & & & & & & - & & & & - \\
\hline Apert. a la experiencia & - & & & & + & & & & & - & & & \\
\hline
\end{tabular}




\section{DISCUSIÓN}

Las características de la muestra se asemejan en lo general a las de otros estudios realizados en centros específicos de atención a drogodependientes (Nadeau et al., 1999; Mestre et al., 2001; Pedrero, 2002) y se diferencian netamente de las que se realizan en servicios de salud mental general (Iglesias et al., 2000), especialmente en la baja proporción de mujeres en los primeros.

En lo que se refiere a la descripción de las características de personalidad de la muestra, en los resultados en el BFO sí existen diferencias entre estudios similares extraídos de poblaciones muy semejantes, como la que se presenta en Pedrero (2002): en el trabajo actual la muestra presenta valores ligeramente más altos en Afabilidad y Tesón y más bajos en Estabilidad Emocional. La razón estriba en un criterio diferente de selección de la muestra, que en aquél estudio era aleatorio y se administraba únicamente a sujetos que demandaban tratamiento, en la primera semana, mientras que el presente estudio se realiza sobre sujetos que se encuentran en diversos momentos del tratamiento en un mismo dispositivo.

En lo que se refiere a los resultados obtenidos con el MCMI-II se obtienen resultados semejantes a los de otros estudios que utilizan la primera versión de este cuestionario (Nadeau et al., 1999) o el propio MCMI-II (Mestre et al., 2001), destacando la alta prevalencia de algunos trastornos y la posibilidad de coexistencia de varios patrones disfuncionales en un mismo sujeto. Mestre y colaboradores concluyen que este cuestionario sobrepuntúa esta patología, aconsejando utilizar complementariamente una entrevista clínica diagnóstica. Nadeau, en cambio, sugiere dos razones a tener en cuenta al interpretar los datos del MCMI: por una parte, este cuestionario no es un instrumento diagnóstico -al menos no enfocado al diagnóstico categorial- y no debe ser utilizado como tal; la intención que guía su elaboración es, precisamente, dar una alternativa a los diagnósticos categoriales y sólo después se ha intentado aproximar un enfoque y otro a partir del establecimiento de tasas base para diferentes poblaciones. Para la obtención de dichas tasas base se ha recurrido a los estudios epidemiológicos que cifran la prevalencia encontrada en cada población. El procedimiento es, en sí mismo, circular, y sólo debe ser tenido en cuenta como aproximación y no como suplantación de métodos que son más apropiados al enfoque categorial. No obstante, sería recomendable revisar las tasas base establecidas en la actualidad -al menos las que se refieren a la población que nos ocupa, los drogodependientes- quizá utilizando muestras mayores que permitieran un mejor ajuste a la concepción categorial, como es la pretensión del autor; o bien recurrir a criterios más restrictivos, como puede ser establecer el punto de corte para la presencia de un trastorno en la TB>84, lo que permitiría una aproximación a la prevalencia estimada mediante métodos no dimensionales. Parte de estos problemas pretenden ser resueltos en la nueva versión del cuestionario, el MCMI-III (Jankowski, 2002), cuyas escalas tienen como referencia las categorías del DSM-IV y se ha procurado limitar el solapamiento entre escalas.

También hay que tener en cuenta algunas limitaciones de las propias categorías diagnósticas cuyos criterios son redundantes, en algunos aspectos, con los que se utilizan para el diagnóstico de abuso y dependencia de sustancias: por ejemplo, la alta prevalencia de Trastorno Antisocial en heroinómanos quizá se deba a que la propia conducta de búsqueda y administración de la sustancia "obliga" a adoptar algunos de los comportamientos que sirven como criterios para el diagnóstico de este desorden (Bolinches et al., 2002); o el hecho de que el consumo de sustancias sea uno de los criterios para formalizar el diagnóstico de Trastorno Límite, otro de los más informados en toxicómanos.

En segundo lugar, Nadeau cuestiona la estabilidad temporal del MCMI: según estudios de su propio equipo (Landry et al., 1996) y otros que cita (Zimmerman, 1994), el estudio test-retest del MCMI, al menos en su primera versión, revela que la intensidad de los síntomas decrece después de unas pocas semanas de tratamiento, no sólo en la sintomatología propia del Eje I, sino también en la que nos ocupa, y atribuye tal decremento a la desaparición de los efectos tóxicos de la sustancia. No obstante, no sería justo atribuir este descenso únicamente a deficiencias del cuestionario o a los efectos fisiológicos de las sustancias, sin advertir que la propia intervención (psicoterapéutica y farmacológica) puede estar modificando determinados aspectos del patrón de conducta desadaptativo, aproximando sus valores a los de la normalidad. Es extremadamente complejo discernir el papel del tóxico, de la medicación prescrita, de la psicoterapia y otros componentes probables en la reducción de los síntomas que afectan al Eje II, como lo es admitir que el constructo de Trastorno de la Personalidad no es tan estable como debiera, estando sometido a tantas fuentes de variabilidad.

Entrando ya en el objetivo de este trabajo -las relaciones apreciadas entre las dimensiones del BFO y las escalas del MCMI-II- los resultados obtenidos en nuestro trabajo se adecuan muy consistentemente con los principios teóricos que guían la caracterización de las escalas en el modelo de Millon. Así, los sujetos esquizoides y evitadores compartirían un fuerte grado de introversión, una muy baja afabilidad, poca perseverancia en las tareas y una nula apertura mental, aunque los evitadores presentarían un mayor grado de inestabilidad emocional, y ambos aparecerían como formas menores del patrón Esquizotípico. Los sujetos 
dependientes serían muy afables, en tanto que esa manera de comportarse les aseguraría la atención de las figuras que proveen la seguridad buscada. Los histriónicos serían sujetos fuertemente extrovertidos en la búsqueda de un refuerzo externo del que dependería permanentemente una estructura emocional extremadamente vulnerable, como en el caso de los narcisistas, aunque éstos no se sienten tan inclinados a ser agradables con aquéllos que deben proveerles del refuerzo que creen merecer. Los antisociales, muy dominantes, incapaces de empatizar con los demás y de comportarse de forma agradable con ellos, serían proclives a las respuestas impulsivas frente a situaciones que implicaran amenaza a su inestable estructura emocional, de forma muy similar a lo que se observa en los dos patrones agresivos, aunque la pasividad se caracteriza por una nula apertura mental; los tres patrones parecen ajustarse adecuadamente a formas menores del patrón Paranoide y también al Límite, aunque éste carece de la dominancia de que hacen gala los anteriores. Los obsesivo/compulsivos se caracterizan por un fuerte tesón en el cumplimiento de sus tareas del que depende su fuerte estabilidad emocional, aunque ésta puede ser también producto de la alta distorsión de sus percepciones.

Estos resultados están, en gran medida, en la misma línea de los encontrados en otros estudios (ver Tabla 12). La concordancia entre muchas de las relaciones observadas hace pensar en la existencia de una estructura sólida; pero para algunos autores, como Pelechano (1996), son precisamente las discrepancias, también muy amplias, las que desacreditan

\begin{tabular}{|c|c|c|c|c|c|c|c|}
\hline 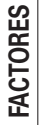 & $\begin{array}{c}\text { Wiggins y Pincus } \\
\text { (1989) } \\
\text { (con normales) }\end{array}$ & $\begin{array}{c}\text { Costa y McCrae } \\
\text { (1990) } \\
\text { (con normales) }\end{array}$ & $\begin{array}{c}\text { Trull (1992) SIDP-R } \\
\text { (con pacientes } \\
\text { psiquiátricos) }\end{array}$ & $\begin{array}{c}\text { Trull (1992) MMPI } \\
\text { (con pacientes } \\
\text { psiquiátricos) }\end{array}$ & $\begin{array}{c}\text { Trull (1992) PDQ-R } \\
\text { (con pacientes } \\
\text { psiquiátricos) }\end{array}$ & $\begin{array}{c}\text { (Brooner (2002) } \\
\text { (con } \\
\text { drogodependientes) }\end{array}$ & $\begin{array}{l}\text { Presente } \\
\text { trabajo }\end{array}$ \\
\hline 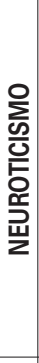 & $\begin{array}{l}\text { + Límite } \\
\text { + Evitación } \\
\text { + Pas/Agre } \\
\text { + Depend } \\
\text { + Compuls } \\
\text { - Narcisista } \\
\text { - Antisocial }\end{array}$ & $\begin{array}{l}\text { + Límite } \\
\text { + Evitación } \\
\text { + Pas/Agre } \\
\text { + Dependiente } \\
\text { + Compuls } \\
\text { - Narcisista } \\
\text { + Antisocial } \\
\text { + Esquizoide } \\
\text { + Esquizotíp } \\
\text { + Paranoide } \\
\text { - Histriónico }\end{array}$ & $\begin{array}{l}\text { + Límite } \\
\text { + Depend } \\
\text { + Obs/Com } \\
\text { + Narcisista } \\
\text { + Histriónico }\end{array}$ & $\begin{array}{l}\text { + Límite } \\
\text { + Evitación } \\
\text { + Pas/Agre } \\
\text { + Dependiente } \\
\text { + Compuls } \\
\text { - Narcisista } \\
\text { + Antisocial } \\
\text { + Esquizotíp } \\
\text { + Paranoide }\end{array}$ & $\begin{array}{l}\text { + Límite } \\
\text { + Evitación } \\
\text { + Pas/Agre } \\
\text { + Dependiente } \\
\text { + Obs/Com } \\
\text { + Narcisista } \\
\text { + Esquizotíp } \\
\text { + Paranoide } \\
\text { + Histriónico }\end{array}$ & $\begin{array}{l}\text { + Límite } \\
\text { + Dependiente } \\
\text { + Paranoide } \\
\text { + Antisocial }\end{array}$ & $\begin{array}{l}\text { + Evitación } \\
\text { + Histriónico } \\
\text { + Antisocial } \\
\text { + Agr/Sád } \\
\text { + Pas/Agre } \\
\text { + Autodest } \\
\text { - Compulsiva } \\
\text { + Esquizotip } \\
\text { + Límite }\end{array}$ \\
\hline 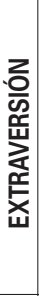 & $\begin{array}{l}\text { + Histriónico } \\
\text { + Narcisista } \\
\text { + Antisocial } \\
\text { - Esquizoide } \\
\text { - Evitación }\end{array}$ & $\begin{array}{l}\text { + Histriónico } \\
\text { + Narcisista } \\
\text { - Esquizoide } \\
\text { - Evitación } \\
\text { + Límite } \\
\text { - Dependiente } \\
\text { - Obs/Com } \\
\text { - Pas/Agre } \\
\text { - Esquizotíp }\end{array}$ & $\begin{array}{l}\text { - Esquizoide } \\
\text { - Evitación } \\
\text { - Obs/Com } \\
\text { - Esquizotíp }\end{array}$ & $\begin{array}{l}\text { + Histriónico } \\
\text { + Narcisista } \\
\text { - Esquizoide } \\
\text { - Evitación } \\
\text { - Obs/Com } \\
\text { - Esquizotíp }\end{array}$ & $\begin{array}{l}\text { + Histriónico } \\
\text { - Esquizoide } \\
\text { - Evitación }\end{array}$ & $\begin{array}{l}\text { - Antisocial } \\
\text { - Evitación } \\
\text { - Paranoide }\end{array}$ & $\begin{array}{l}\text { - Esquizoide } \\
\text { - Evitación } \\
\text { - Esquizotíp } \\
\text { + Histriónico } \\
\text { + Narcisista } \\
\text { + Agr/Sád } \\
\text { + Paranoide }\end{array}$ \\
\hline 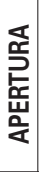 & + Esquizotíp & $\begin{array}{l}\text { + Histriónico } \\
\text { + Antisocial }\end{array}$ & - Esquizoide & $\begin{array}{l}\text { + Histriónico } \\
\text { + Narcisista } \\
\text { - Esquizoide }\end{array}$ & $\begin{array}{l}\text { - Esquizoide } \\
\text { + Límite }\end{array}$ & & $\begin{array}{l}\text { - Esquizoide } \\
\text { - Evitación } \\
\text { - Pasivo/Agres } \\
\text { - Autodestruct } \\
\text { - Esquizotípico }\end{array}$ \\
\hline 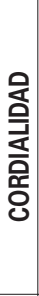 & $\begin{array}{l}\text { + Dependien } \\
\text { - Antisocial } \\
\text { - Paranoide } \\
\text { - Narcisista }\end{array}$ & $\begin{array}{c}\text { + Dependiente } \\
\text { - Antisocial } \\
\text { - Paranoide } \\
\text { - Narcisista } \\
\text { - Esquizoide } \\
\text { - Obs/Com } \\
\text { - Pas/Agre } \\
\text { - Esquizotíp } \\
\text { - Límite }\end{array}$ & $\begin{array}{l}\text { - Antisocial } \\
\text { - Paranoide } \\
\text { - Narcisista } \\
\text { - Esquizoide } \\
\text { - Obs/Com } \\
\text { - Pas/Agre } \\
\text { - Esquizotíp } \\
\text { - Límite }\end{array}$ & $\begin{array}{l}\text { - Dependiente } \\
\text { - Paranoide } \\
\text { - Pas/Agre } \\
\text { - Esquizotíp } \\
\text { - Límite }\end{array}$ & $\begin{array}{l}\text { - Antisocial } \\
\text { - Paranoide } \\
\text { - Narcisista } \\
\text { - Esquizoide } \\
\text { - Compuls } \\
\text { - Pas/Agre } \\
\text { - Esquizotíp } \\
\text { - Límite }\end{array}$ & $\begin{array}{l}\text { - Antisocial } \\
\text { - Paranoide }\end{array}$ & $\begin{array}{l}\text { + Dependiente } \\
\text { - Antisocial } \\
\text { - Paranoide } \\
\text { - Agres/Sád }\end{array}$ \\
\hline 을 & $\begin{array}{l}\text { + Compuls } \\
\text { - Antisocial } \\
\text { - Pas/Agre }\end{array}$ & $\begin{array}{l}\text { - Antisocial } \\
\text { - Pas/Agre } \\
\text { + Esquizoide } \\
\text { - Dependiente } \\
\text { - Histriónico } \\
\text { - Límite } \\
\text { - Paranoide }\end{array}$ & $\begin{array}{l}\text { - Antisocial } \\
\text { - Límite }\end{array}$ & $\begin{array}{l}\text { - Antisocial } \\
\text { - Pas/Agre } \\
\text { - Dependiente }\end{array}$ & $\begin{array}{l}\text { - Antisocial } \\
\text { - Pas/Agre } \\
\text { - Dependiente } \\
\text { - Narcisista }\end{array}$ & - Antisocial & $\begin{array}{c}\text { + Compulsiva } \\
\text { - Paranoide } \\
\text { - Límite }\end{array}$ \\
\hline
\end{tabular}


el modelo. La dimensión que aparece más consistentemente relacionada con la presencia de trastornos es la de Neuroticismo o Inestabilidad Emocional, implicada en la mayor parte de los trastornos, aunque en algún caso, como en el del Trastorno Narcisista, unas veces en sentido negativo y otras en sentido positivo. El Tesón se halla habitualmente implicado en el Trastorno Antisocial y, en sentido contrario, en el Obsesivo/Compulsivo. La Introversión, o baja Energía, se relaciona consistentemente con los trastornos Esquizoide y Fóbico, pero de manera variable con los demás. La Afabilidad se relaciona con el Trastorno Paranoide. La Apertura Mental apenas presenta relaciones con los trastornos, siendo frecuente, pero no unánime, su relación con el trastorno Esquizoide.

La variedad de resultados entre diversos estudios puede deberse a diversos factores: (1) la inadecuación del modelo de 5 factores, (2) la inapropiada definición de los trastornos, (3) deficiencias o diferencias entre los instrumentos diagnósticos, (4) diferentes momentos para la realización de las pruebas, etc. La primera cuestión sigue abierta; existen argumentos que apoyan el modelo de 5 factores: es un modelo que aparece consistentemente en multitud de estudios, se obtiene mediante un procedimiento matemático, empieza a contar con evidencias de estar en posesión de un soporte genético e incluso se encuentra su relación con algunas moléculas y su mecanismo de acción en la modulación de los estados de ánimo (Lesch et al., 1996). Pero también existen críticas de importancia: para algunos sigue siendo sólo un artefacto matemático, el hallazgo de 5 factores no garantiza que sean suficientes para explicar los patrones complejos de conducta, ni que todos los factores tengan la misma importancia en su caracterización; de hecho, muchos investigadores siguen buscando el sexto y sucesivos factores en un intento por completar lo que se tiene por estructura inacabada de la personalidad (Ashton et al., 2000; Saucier, 2001) o el hallazgo de factores de orden superior de composición estrictamente ambiental (Jang et al., 2001). Hay que añadir a estas críticas la que hace referencia a una cuestionable independencia de los pretendidamente independientes cinco factores, como se observa en las amplias correlaciones observadas tanto en la muestra normativa española del BFQ como las que aparecen en nuestro estudio (ver Tabla 4), aún más fuertes y significativas que en aquélla.

El modelo categorial, a su vez, cuenta con un amplio consenso y un cuerpo de investigación mucho más amplio que el otro modelo, también cuenta con soporte en hallazgos psicobiológicos (Carrasco y Díaz, 1997; Besteiro et al., 2002) y es aceptado incluso por los investigadores del modelo de cinco factores, al menos en su concepción definicional (Widiger, 1993). Sin embargo, en su contra se emiten críticas de gran calado, como que su formulación se realiza a partir de descripciones fenomenológicas procedentes de muy diversos enfoques teóricos, las técnicas de diagnóstico clínico no presentan adecuadas tasas de fiabilidad, el estudio matemático no confirma los clusters que predice el modelo, no existe acuerdo en cuántos pueden ser los trastornos, etc. (Westen y Shedler, 2000).

En cuanto a los instrumentos elaborados desde el enfoque dimensional, el NEO-PI-R es una escala con excelentes propiedades psicométricas y ampliamente utilizado en la investigación y la clínica. El BFQ, utilizado en el presente trabajo, no posee tanta difusión, quizá por su procedencia ajena al ámbito norteamericano predominante, aunque acaso también porque no ha conseguido demostrar una superioridad sobre aquél. En cuanto al MCMI, se trata de un cuestionario extremadamente complejo, tanto para su utilización clínica como con fines de investigación; basado en el modelo estructural politético, asume el solapamiento entre escalas, puesto que no requiere su independencia, lo que refuerza, en contrapartida, la consistencia interna de cada dimensión. Quizá tenga su principal limitación en la inevitable dependencia del modelo categorial para la caracterización de los trastornos que pretende medir dimensionalmente. Estudios futuros, basados en muestras mucho más amplias, quizá permitan una caracterización nueva de los patrones en función de los diversos perfiles dimensionales sin tener que recurrir a las categorías de las que huye el modelo en que se fundamenta.

Por último, la cuestión de la estabilidad temporal parece resuelta en el caso de los instrumentos del modelo de 5 factores, pero no en el caso del MCMI-II: si los patrones que ofrece no son estables, es cuestionable que se trate de perfiles de personalidad y acaso se superpongan elementos que tienen que ver con el Eje I, originando una morbilidad espuria en el Eje II. Hay que considerar que en los estudios de estabilidad temporal de la anterior versión del MCMI, los estudios con drogodependientes eran los que proporcionaban cifras más bajas a 1 mes (McMahon et al., 1985), pero no es admisible que en ese periodo se puedan apreciar cambios en los patrones de personalidad.

Lo que propone el modelo de 5 factores no es prescindir del modelo categorial, sino caracterizar los patrones de conducta desadaptativa a partir de unidades más básicas, para, en un paso posterior, sustituir las actuales categorías por otras que presenten mayor fiabilidad y validez, eliminando los solapamientos. Un trastorno de personalidad se definiría, entonces, no por sus manifestaciones fenomenológicas, sino por su peculiar perfil de combinación de los elementos básicos. Si se profundiza en las bases biológicas de las dimensiones, en los determinantes ambientales que las modulan, en las combinaciones posibles y en las manifestaciones conductuales, entonces sí podríamos contar con una adecuada caracterización de tales patrones de conducta compleja (Widiger et al., 2002a). 
La cuestión se complica enormemente cuando además las personas utilizan sustancias. Porque las drogas son elementos ambientales que modifican la expresión de los sustratos biológicos en la fase de formación de la personalidad, de modo que, parafraseando a Meyer (1986), la personalidad puede ser factor determinante en el inicio del consumo de drogas, o éstas ser las que modifiquen la personalidad hacia patrones desadaptativos, o actuar como moduladores de rasgos previos, o ser independientes unos y otros. El hecho de que las sustancias autoadministradas utilicen las mismas vías neuronales que se encuentran implicadas en la génesis de los rasgos básicos de la personalidad (Roques, 2000; Fernández-Espejo, 2002) justifica la existencia de una gran comorbilidad (Cervera et al., 2001), sin que puedan obviarse todos los aspectos ambientales no directamente relacionados con los efectos químicos de la sustancia. De hecho, las formulaciones actuales de la adicción se describen mejor como modelos de diátesis-estrés bioconductuales, considerándose que el comienzo y el curso de la adicción derivan de una interacción recíproca continua entre las vulnerabilidades biológicas y psicológicas, y los recursos del individuo y sus circunstancias psicosociales (Fernández Miranda, 2002). Lo cual sugiere una amalgama de circunstancias que imposibilita conocer -en la investigación, la clasificación y la clínica- qué parte de patología hay que adjudicar a la sustancia y que parte a la personalidad del individuo, cuál de todos los aspectos debe ser priorizado en el tratamiento, cuáles de los síntomas que manifiesta el sujeto desaparecerán si se revierte el hábito y cuáles persistirán, qué cambios irreversibles se habrán producido en sus estructuras y funciones biológicas y cómo se expresarán tales modificaciones en su conducta observable y encubierta, qué circunstancias ambientales seguirán operando como estímulos discriminativos para poner en marcha mecanismos biológicos y viceversa.

En todo caso, sí comprendemos en los últimos años que en la conducta de un sujeto que demanda tratamiento por consumo de drogas hay aspectos que se pueden modificar (farmacológica y psicoterapéuticamente) y otros que hay que considerar invariables (como la alta reactividad emocional, p.e.) y que deben ser tenidos en cuenta a la hora de formular programas de tratamiento. Contrariamente a lo que ha sido la característica principal en los años anteriores, la última década ha empezado a desplazar el foco de atención, en los programas de tratamiento de drogodependientes, desde la droga, como elemento unificador de quienes solicitaban tratamiento, hacia las características personales de los sujetos que las consumen. Si en los 80 los programas de tratamiento focalizaban su atención en los sujetos heroinómanos y, más tarde, en los cocainómanos o en los consumidores de drogas de síntesis, desde hace algunos años empieza a cobrar cuerpo el enfoque de la droga como manifestación de alguna característica disfuncional de los individuos que se las autoadministran y propugnando, en consonancia, la diversificación de perfiles de sujetos en función de características personales y no de la droga consumida, cuyo interés se reduce a la funcionalidad que cumple con relación a las dificultades personales. Tales enfoques no tienen por qué desembocar en una consideración meramente medicalista, sujeta al concepto de enfermedad mental (Marina, 1999, 2001), sino a la correcta aplicación de un completo enfoque biopsicosocial. Reducir las conductas de adicción a sustancias a una mera disfunción biológica es un error tan grande como prescindir de los conocimientos de la biología, la farmacología y la medicina para su resolución. La consideración del drogodependiente, y de quien tiene un padecimiento psicológico, como enfermos puede tener una justificación protectora de cara a la sociedad, en la medida en que absuelve de responsabilidad sobre sus actos y permite una consideración que de otro modo dificultaría el abordaje de estos problemas, pero esto está alejado del quehacer científico (Lemos, 1995) y dificulta la aplicación de técnicas complejas, como la psicoterapia, que requieren la implicación activa del sujeto en la promoción de cambios de conducta, las expectativas de que puede cambiar su situación y de que tal cambio le va a proporcionar un cierto bienestar, y la responsabilización de sus actos que es, en definitiva, lo que puede permitir un desenvolvimiento autónomo en su medio social de referencia.

En el extremo opuesto, aplicar cualquier modalidad de psicoterapia sin prestar atención a los aspectos invariantes del sujeto, de índole biológica, o de las manifestaciones conductuales de rasgos estables de la personalidad o de patrones desadaptativos de comportamiento, puede abocar a un fracaso que no sólo daña al paciente sino al propio profesional que intenta promover un cambio imposible. La búsqueda de apoyos para ese tratamiento a veces obliga a depositar parte del control sobre el proceso en agentes como los familiares, sin tener en cuenta que, ya fuere por factores hereditarios o ambientales, un trastorno de personalidad suele darse en un entorno poco estructurado en el cual los familiares presentan, a su vez, trastornos de igual o superior entidad a los del drogodependiente (Peña, 2002).

La vía abierta por el estudio del modelo de 5 factores y su relación con los desórdenes de conducta puede favorecer la adopción de enfoques más adecuados en la clínica. Las estrategias ya bien conocidas en el ámbito de las drogodependencias, como la prevención de recaídas, el entrenamiento asertivo, en habilidades sociales, en afrontamiento del estrés, los enfoques educativos y, por supuesto, el abordaje farmacológico, pueden beneficiarse de un mejor conocimiento de los aspectos básicos de la conducta y la 
manera en que pueden entrelazarse y manifestarse. No sería descabellado pensar que, en pocos años, los sujetos fueran atendidos selectivamente en función de las necesidades estimadas a partir de sus rasgos de personalidad, de modo que, por ejemplo, un paciente con pronunciada inestabilidad emocional, recibiera los fármacos adecuados para su modulación en tanto acudía a grupos específicos de terapia enfocada al control emocional; grupo al que no tendrían por qué acudir personas con adecuados recursos en esta dimensión pero, por ejemplo, un bajo tesón y una baja apertura mental, que serían abordados desde grupos específicos de terapia ocupacional y educativos. Paralelamente ambos realizarían actividades, individuales o grupales, encaminadas a la prevención de recaídas que, sin embargo, conocerían de antemano los límites a los que se podría llegar y no tendrían como única meta la abstinencia, sino unos objetivos adecuados a las capacidades y limitaciones de cada individuo en particular. Sirva este apunte como esbozo de un cambio de orientación en la terapia en la medida en que atienda más a las características del sujeto y menos a elementos inertes como la sustancia.

\section{REFERENCIAS BIBLIOGRÁFICAS}

ASHTON, M.C.; LEE, K.; SON, C. (2000): Honesty as the Sixth Factor of Personality: correlations with Machiavellanism, Primary Psychopathy, and Social Adroitness. European Journal of Personality, 14 (359368).

AVIA, M.D.; SANZ, J.; SÁNCHEZ-BERNARDOS, M.L.; MARTÍNEZ ARIAS, M.R.; SILVA, F.; GRAÑA, J.L. (1995): The five-factor model. Relations of the NEO-PI with other personality variables. Personality and Individual Differences, 19 (1): 81-97.

BALL, A. (2002): Big Five, alternative five, and seven personality dimensions. Validity in substance dependents patients. En P.T.COSTA Jr. y A.WIDIGER (Eds.): Personality disorders and the Five-Factor model of personality ( $2^{\mathrm{a}}$ Ed.): Washington: American Psychological Association.

BERROCAL, C.; ORTIZ-TALLO, M.; FIERRO, A.; JIMÉNEZ, J.A. (2001): Variables clínicas y de personalidad en adictos a heroína. Anuario de Psicología, 32(1): 67-87.

BESTEIRO GONZÁLEZ, J.L.; LEMOS GIRÁLDEZ, S.; MUÑIZ FERNÁNDEZ, J. (2002): Evaluación de las funciones córtico-orbitales frontales y de las características psicofisiológicas en los trastornos de la personalidad del DSM-IV. Actas Españolas de Psiquiatría, 30 (1): 54-62.

BLASHFIELD, R.K. (1986): Structural approaches to classification. En T.Millon y G.L.Klennan (Eds.): Contemporary directions in psychopathology. Nueva York: Guilford Press.
BLUME, S.B. (1989): Dual diagnosis: psychoactive substance dependence and the personality disorders. Journal of Psychoactive Drugs, 21: 139-144.

Bolinches F, De Vicente P, Castellano Gómez M, Pérez-Galvez B, Haro G, Martinez-Raga J, Cervera G (2002): Personalidades impulsivas y trastornos por uso de sustancias: algo más que un diagnóstico dual. Trastornos Adictivos, 4 (4): 216-222.

BORKENAU, P.; OSTENDORF, F. (1993): NEO-Fünf-FaktorenInventar (NEO-FFI) nach Costa und McCrae. Góttingen: Hogrefe.

BORKENAU, P.; RIEMANN, R.; ANGLEITNER, A.; SPINATH, F.M. (2001): Genetic and environmental influences on observed personality: evidence from the german observational study of adult twins. Journal of Personality and Social Psychology, 80 (4): 655-668.

BROONER, R.K.; SCHMIDT, C.W.JR.; HERBST, J.H. (2002): Personality trait characteristics of opioid abusers with and without comorbid personality disorders. En P.T.COSTA Jr. y A.WIDIGER (Eds.): Personality disorders and the Five-Factor model of personality (2' Ed.): Washington: American Psychological Association.

BROONER, R.K.; HERBST, J.H.; SCHMIDT, C.W.; BIGELOW, G.E.; COSTA, P.T.JR. (1993): Antisocial personality disorder among drug abusers: Relations to other personality diagnoses and the five-factor model of personality. Journal of Nervous and Mental Disease, 181: 313-319.

BROONER, R.K.; KING, V.L.; KIDORF, M.; SCHMIDT, C.W.; BIGELOW, G. E. (1997): Psychiatric and substance use comorbidity among treatment-seeking opioid users. Archives of General Psychiatry, 54: 71-80.

CALSYN, D.A.; FLEMING, C.; WELLS, E.A.; SAXON, A.J. (1996): Personality disorder subtypes among opiate addicts in methadone maintenance. Journal of Addictive Diseases, 10: 3-8.

CAPRARA, G.V.; BARBARANELLI, C.; BORGOGNI, L.; PERUGINI, M. (1993): The "BigFive Questionnaire": A new questionnaire to assess the five factor model. Personality and Individual Differences, 15, 281-288.

CAPRARA, G.V.; PERUGINI, M. (1994): Personality describes by adjectives: The generalizability of the BigFive to the Italian lexical context. European Journal of Personality, 8 (5): 357-369.

CAPRARA, G.V.; BARBARANELLI, C.; BORGOGNI, L. (1995): BFQ. Cuestionario "Big Five."Versión española de J. Bermúdez. Madrid: TEA Ediciones.

CARRASCO, J.L.; DÍAZ, M. (1997): Psicobiología de los trastornos de la personalidad. Psiquiatría. COM [revista electrónica] Septiembre [citado 28 de agosto 2002]; 1(3): Disponible en URL: http://www.psiquiatria.com/ psiquiatria/vol1 num3/art_6.htm.

CERVERA， G.; VALDERRAMA， J.C.; BOLINCHES, F.; MARTÍNEZ, J. (1997): Pauta de desintoxicación frente a estabilización y mantenimiento con metadona en adictos a opiáceos con trastorno de la personalidad. Psiquiatría Biológica, 5 1999 ; 4: 181-186. 
CERVERA MARTÍNEZ, G.; RUBIO VALLADOLID, G.; HARO CORTÉS, G.; BOLINCHES CLARAMONTES, F.; DE VICENTE MANZANARO, P.; VALDERRAMA ZURIÁN, J.C. (2001): La comorbilidad entre los trastornos del control de los impulsos, los relacionados con el uso de sustancias y los de la personalidad. Trastornos Adictivos, 3 (1): 3-10.

CLONINGER, C.R.; SVRAKIC, D.M.; PRZYBECK, T.R. (1993): A psychobiological model of temperament and character. Archives of General Psychiatry, 50: 975-990.

COSTA, P.T. JR.; McCRAE, R.R. (1985): NEO Personality Inventory manual. Odessa: Psychological Assessment Resources.

COSTA, P.T. JR.; McCRAE, R.R. (1988): Personality in adulthood: a six-year longitudinal study of self reports and spouse ratings on the NEO Personality Inventory. Journal of Personality and Social Psychology, 54: 853863.

COSTA, P.T. JR.; McCRAE, R.R. (1990): Personality disorders and the five-factor model of personality. Journal of Personality Disorders, 4: 362-37 1.

COSTA, P.T. JR.; McCRAE, R.R. (1992): Revised NEO Personality Inventory (NEO-PI-R) and NEO Five-Factor Inventory (NEO-FFI) professional manual. Odessa: Psychological Ássessment Resources.

CRAIG, R.J.; WEIBERG, D. (1992): Assessing drug abusers with the Millon Clinical Multiaxial Inventory: a review. Journal of Substance Abuse Treatment, 9:249-255.

DEJONG, C.A.J.; VAN DEN BRINK, W.; HARTEVELD, F.H. (1993): Personality disorders in alcoholics and drug addicts. Comprehensive Psychiatry, 34 (2): 87-94.

DE RAAD, B. (1994): An expedition in search of a fifth universal factor: key issues in the lexical approach. European Journal of Personality, 8 (4): 229-250.

FERNÁNDEZ-ESPEJO, E. (2002): Bases neurobiológicas de la drogadicción. Revista de Neurología, 34 (7): 659-664.

FERNÁNDEZ MIRANDA, J.J. (2002): Trastornos de personalidad y adicción: relaciones etiológicas y consecuencias terapéuticas. Anales de Psiquiatria, 18 (9): 421-427.

FLYNN, P.M.; CRADDOCK, S.G.; LUCKEY, J.W.; HUBBARD, R.L.; DUNTEMAN, G.H. (1996): Comorbidity of antisocial personality and mood disorders among psychoactive substance dependent treatment clients. Journal of Personality Disorders, 10 (1): 56-67.

FLYNN, P.M.; McCANN, J.T.; FAIBBANK, J.A. (1995): Issues in the assessment of personality disorder and substance abuse using the Millon Clinical Multiaxial Inventory (MCMI-II): Journal of Clinical Psychology, 51(3): 415-2 1

GARCÍA LÓPEZ, A.; EZQUIAGA TERRAZAS, E. (1992): Psicopatología asociada al consumo de drogas. Actas Luso-Españolas de Neurología, Psiquiatría y Ciencias Afines, 20 (4): 175-187.

HALIKAS, J.A.; CROSBY, R.D.; PEARSON, V.L.; NUGENT, S.M.; CARLSON, G.A. (1994): Psychiatric comorbidity in treatment-seeking cocaine abusers. American Journal on Addictions, 3: 25-35.
HIRSCHFELD, R.M.A. (1993): Personality disorders: Definitions and diagnosis. Journal of Personality Disorders, 7(Supl): 9-18.

HOGAN, R. (1987): Personality Psichology: back to basics. En J.ARONOFF, A.RABIN y R.ZUCKER (Eds.): The emergence of personality. Nueva York: Springer.

IGLESIAS GARCÍA, C.; GIMENO TEJEDOR, A.; DÍAZ CONESAL, I.; INDA CARO, M. (2000): Evaluación dimensional de trastornos de personalidad definidos categorialmente, cinco factores vs. clasificación DSM-IV. Estudio Piloto. Actas Españolas de Psiquiatría, 28 (2): 71-76.

JANG, K.L.; VERNON, P.A.; LIVESLEY, W.J. (2001): Behavioural-Genetic perspectives on personality function. Canadian Journal of Psychiatry, 46: 234-244.

Jankowski, D. (2002): A Beginner's Guide to the MCMI-III. Washington DC: American Psychological Association.

KOKKEVI, A.; STEFANIS, C. (1995): Drug- abuse and psychiatric comorbidity. Comprehensive Psychiatry, 36 (5): 329-337.

KOSTEN, T.A.; KOSTEN, T.R.; ROUNSAVILLE, B.J. (1989): Personality disorders in opiate addicts show prognostic specifity. Journal of Substance Abuse Treatment, 6: 163-168.

KOSTEN, T.R.; ROUNSAVILLE, B.J. (1986): Psychopathology in opioid addicts. Psychiatric Clinics of North America, 9 (3): 515-532.

KRANZLER, H.R.: SATEL, S.; APTER, A. (1994): Personality disorders and associated features in cocainedependent inpatients. Comprehensive Psychiatry, 35: 335-340.

LANDRY, M.; NADEAU, L.; RACINE, S. (1996): La prévalence des troubles de la personnalité dans huit centres de réadaptation du Québec. Cahier de recherche. Montreal: Recherche et Intervention sur les Substances Psychoactives.

LEMOS, S. (1995): Clasificación y diagnóstico en psicopatología. En A.BELLOCH, B.SANDÍN y F.RAMOS (Coord.): Manual de Psicopatología, vol 1. Madrid: McGraw Hill.

LESCH, K.P.; BENGEL, D.; HEILS, A.; ZHANG, S.; GREENBURG, B.D.; PETRI, S. et al. (1996): Association of anxiety-related traits with a polymorphism in the serotonin transporter gene regulatory region. Science, 274: 1527-1530.

LEWIS-FERNANDEZ, R.; KLEINMAN, A. (1995): Cultural psychiatry: Theoretical, clinical, and research issues. Psychiatric Clinics of North America, 18 (3): 433-48.

LIVESLEY, W.J. (2000): Introduction: Critical issues in the classification of personality disorders. Journal of Personality Disorders, 14: 1-2.

MARINA, P. (1999): ¿Qué nos han enseñado los estudios de seguimiento de toxicómanos? Adicciones, 11 (3): 237241.

MARINA GONZÁLEZ, P.A. (2001): ¿Es posible un tratamiento diferente de las adicciones? Revista de la Asociación Española de Neuropsiquiatría, 12 (79): 9-16.

MARLOWE, D.B., HUSBAND, S.D.: LAMB, R.J.; KIRBY, K.C.: IGUCHI, M.Y.; PLATT, J. J. (1995): Psychiatric comorbidity in cocaine dependence. Diverging trends, 
Axis II spectrum, and gender differentials. American Journal on Addictions, 4: 70-81.

McCRAE, R.R.; JANG, K.L.; LIVESLEY, W.J.; RIEXUNN, R.; ANGLEITNER, A. (2001): Sources of structure: genetic, environmental, and artifactual influences on the covariation of personality traits. Journal of Personality, 69 (4): 511-535.

McMAHON, R.C.; FLYNN, P.; DAVIDSON, R. (1985): Stability of the personality and symptom scales of the Millon Clinical Multiaxial Inventory. Joumal of Personality Assessment, 49: 231-234.

McMAHON, R.C.; RICHARDS, S.K. (1996): Profile Patterns, consistency, and change in the Millon Clinical Multiaxial Inventory-II in cocaine abusers. Journal of Clinical Psychology, 52(1): 75-9.

MEEHL, P.E. (1989): Schizotaxia revisited. Archives of General Psychiatry, 46: 935-944.

MESTRE, L.; RISCO, P.; CATALÁN, A.; IBARRA, 0. (2001): Perfiles de personalidad Millon: comparación de pacientes adictos a opiáceos y a cocaína. Trastornos Adictivos, 3 (4): 288-289.

MEYER, R.E. (1986): How to understand the relationship between psychopathology and addictive disorders: another example of the chicken and the egg. En R.E. Meyer (Ed.): Psychopathology and Addictive Disorders. Nueva York: Guilford Press.

MILLON, T. (1999): MCMI-II. Inventario clínico multiaxial de Millon-II. Manual. Adaptación española de A. ÁVILAESPADA (Dir.), F. JIMÉNEZ GÓMEZ (Coord.) y cols. Madrid: TEA.

MILLON, T.; DAVIES, R.D. (1998): Trastornos de la personalidad.- Más allá del DSM-IV. Barcelona:Masson.

NADEAU, L.; LANDRY, M.; RACINE, S. (1999): Prevalence of personality disorders among clients in treatment for addiction. Canadian Journal of Psychiatry, 44 (6): 592596.

NATHAN, P.E.; LANGENBUCHER, J.W. (1999): Psychopathology: description and classification. Annual Review of Psychology, 50:79-107

NELSON-GRAY, R.O. (1 99-1): DSM-IV: Empirical guidelines from psychometrics. Journal of Abnormal Psychology, 100: 308-315.

OLDHAM, J.M.; SKODOL, A.E. (2000): Charting the future of Axis II, Journal of Personality Disorders, 14 (1): 1729.

PEDRERO PÉREZ, E.J. (2002): Estudio de la personalidad de drogodependientes mediante el Big Five Questionnaire. Trastornos Adictivos, 4 (3): 138-150.

PELECHANO, V.; DE MIGUEL, A.; HERNÁNDEZ, M. (1995): Trastornos de Personalidad. En A.BELLOCH, B.SANDÍN y F.RAMOS: Manual de Psicopatología, vol 1. Madrid: McGraw Hill.

PEÑA RODRÍGUEZ, C. (2002): Estudio de conflictos psicológicos en los padres de adictos. Los trastornos de personalidad compulsivo, esquizoide y dependiente. Algunas implicaciones para la terapia y la prevención. Itaca, 6 (2): 33-62.
PLOMIN, R.; DEFRIES, J.C.; McCLERAN, G.E.; RUTTER, M. (1997): Behavioral genetics. New York: Freeman.

REGIER, D.A.; FARMER, M.E.; RAE, DS.; LOCKE, B.Z.; KEITH, B.J.; JUDD, L.L.; GODWIN, F.K. (1 990): Comorbidity of mental health disorders with alcohol and other drug abuse, Journal of the American Medical Association, 264: 2511-2518.

ROQUES, B.P. (2000): La dangerosité des drogues: mécanismes neurobiologiques des addictions et approaches thérapeutiques. Les Sélections de MédecinelSciences, 18: 24-32.

SÁNCHEZ, E.; TOMÁS, V.; CLIMENT, A. (1999): Trastornos de la personalidad en adictos a opiáceos. Adicciones, 11 (3): 221-227.

SANCHEZ BERNARDOS, M.L. (1995): Los trastornos de la personalidad y el modelo de los Cinco Factores: relaciones empíricas. Clínica y Salud, 6(2): 175-188.

SAN NARCISO, G.I.; GUTIÉPREZ, E.; SÁIZ, P.A.; GONZÁLEZ, M.P.; BASCARÁN, M.T.; BOBES, J. (2000): Evaluación de trastornos de la personalidad en pacientes heroinómanos mediante el Intemational Personality Disorders Examination (IPDE): Adicciones, 12 (1): 4356.

SANTOS DIEZ, P.; FORCADA CHAPA, R.; ZAMORANO GARCIA, M. C. (2001): Trastornos de personalidad en alcohólicos. Trastornos Adictivos, 3 (4): 297-298.

SAUCIER, G. (2001): Gone too far - Or not far enough? Comments of the article by Ashton and Lee (2001): European Journal of Personality, 16: 55-62.

TRIANDIS, H. C.; EUNKOOK, M.S. (2002): Cultural influences on personality. Annual Review of Psychology, 53:133-160.

TRULL, T.J. (1992): DSM-111-R. Personality disorders and the five-factor model of personality: an empirical comparison. Journal of Abnormal Psychology, 3: 553560.

TRULL, T.J., McCRAE, R.R. (2002): A Five-Factor perspective on personality research. En P.T. COSTA Jr. Y A.WIDIGER (Eds.): Personality disorders and the FiveFactor model of personality (2" Ed.): Washington: American Psychological Association.

TYRER, P.; CASEY, P.; FERGUSON, B. (1993): Personality disorder in perspective. En P.Tyrer y G.STEIN (Eds.): Personality Disorder Reviewed. Londres: Gaskell, Royal College of Psychiatrists.

WESTEN, D.; SHEDLER, J. (2000): A prototype matching approach to diagnosing personality disorders: Toward DSM-V. Journal of Personality Disorders, 14 (2): 109126.

WIDIGER, T.A. (1993): The DSM-III-R. Categorial personality disorder diagnoses: a critique and an alternative. Psychological Inquiry, 4: 13 5-14 1.

WIDIGER, T.A. (2000): Personality disorders in the $21 \mathrm{st}$ century. Journal of Personality Disorders, 14: 3-16.

WIDIGER, T.A.; COSTA, P.T.JR., McCRAE, R.R. (2002a): A proposal for Axis II: Diagnosing personality disorders using the Five-Factor Model. En P.T. COSTA Jr. y A.WIDIGER (Eds.): Personality disorders and the Five- 
Factor model of personality (2a Ed.): Washington: American Psychological Association.

WIDIGER, T.A.; FRANCES, A.J. (2002b): Toward a dimensional model for the personality disorders. En P.T. COSTA Jr. y A.WIDIGER (Eds.): Personality disorders and the Five-Factor model of personality (2aEd.): Washington: American Psychological Association.

WIDIGER, T.A.; SANDERSON, C.J. (1995): Assessing personality disorders En J.N.BUTCHER (Ed.): Clinical personality assessment: practical approaches. Nueva York: Oxford University Press.

WIDIGER, T.A.; TRULL; T.J.; CLARKIN, J.F.; SANDERSON, C.; COSTA, P.T. (1994): A description of the DSM-III-R and DSM-IV personality disorders with the five-factor model of personality. En P.T. COSTA y T.A. WIDIGER (Eds.): Personality disorders and the five-factor model of personality. Washington: American Psychological Association.
WIDIGER, T.A.; TRULL; T.J.; CLARKIN, J.F.; SANDERSON, C.; COSTA, P.T. (2002c): A description of the DSM-IV personality disorders with the five-factor model of personality. En P.T. COSTA Jr. y A.WIDIGER (Eds.): Personality disorders and the Five-Factor model of personality (2 ${ }^{\mathrm{a}}$ Ed.): Washington: American Psychological Association.

WIGGINS, J.S.; PINCUS, A.L. (1989): Conceptions of personality disorders and dimensions of personality. Psychological Assessment: A Journal of Consulting and Clinical Psychology, 305-316.

WIGGINS, J.S.; PINCUS, A.L. (1992): Personality: structure and assessment. Annual Review of Psychology, 43: 473-504.

ZIMMERMAN, M. (1994): Diagnosing personality disorders: a review of issues and research methods. Archives of General Psychiatry, 51: 225-245. 